\title{
The dam-break problem for concentrated suspensions of neutrally buoyant particles
}

\author{
C. Ancey $\dagger$, N. Andreini and G. Epely-Chauvin $\ddagger$ \\ Environmental Hydraulics Laboratory, École Polytechnique Fédérale de Lausanne, \\ 1015 Lausanne, Switzerland
}

(Received 29 May 2012; revised 15 February 2013; accepted 12 March 2013)

This paper addresses the dam-break problem for particle suspensions, that is, the flow of a finite volume of suspension released suddenly down an inclined flume. We were concerned with concentrated suspensions made up of neutrally buoyant non-colloidal particles within a Newtonian fluid. Experiments were conducted over wide ranges of slope, concentration and mass. The major contributions of our experimental study are the simultaneous measurement of local flow properties far from the sidewalls (velocity profile and, with lower accuracy, particle concentration) and macroscopic features (front position, flow depth profile). To that end, the refractive index of the fluid was adapted to closely match that of the particles, enabling data acquisition up to particle volume fractions of $60 \%$. Particle migration resulted in the blunting of the velocity profile, in contrast to the parabolic profile observed in homogeneous Newtonian fluids. The experimental results were compared with predictions from lubrication theory and particle migration theory. For solids fractions as large as $45 \%$, the flow behaviour did not differ much from that of a homogeneous Newtonian fluid. More specifically, we observed that the velocity profiles were closely approximated by a parabolic form and there was little evidence of particle migration throughout the depth. For particle concentrations in the 52-56\% range, the flow depth and front position were fairly well predicted by lubrication theory, but taking a closer look at the velocity profiles revealed that particle migration had noticeable effects on the shape of the velocity profile (blunting), but had little impact on its strength, which explained why lubrication theory performed well. Particle migration theories (such as the shear-induced diffusion model) successfully captured the slow evolution of the velocity profiles. For particle concentrations in excess of $56 \%$, the macroscopic flow features were grossly predicted by lubrication theory (to within $20 \%$ for the flow depth, $50 \%$ for the front position). The flows seemed to reach a steady state, i.e. the shape of the velocity profile showed little time dependence.

Key words: gravity currents, lubrication theory, suspensions

\section{Introduction}

Suspension flows are important in a variety of engineering and scientific problems, such as the handling and processing of slurries, the pumping of concrete, blood

$\dagger$ Email address for correspondence: christophe.ancey@epfl.ch

$¥$ Sadly, Gaël Epely-Chauvin died in a diving accident during the writing of this paper. 
circulation and debris flows on mountain slopes (Ancey 2012). A distinctive feature of these flows is the migration of particles from regions of high to low shear. Combined with normal stress differences and the coupling between particle arrangement and bulk flow, the resulting locally varying viscosity promotes the development of a nonNewtonian rheology (Stickel \& Powell 2005; Morris 2009). Several migration models have been proposed over the last 20 years to study the particle flux in suspension flows. These models can describe the steady-state concentration and velocity profiles in simple flow geometries (viscometric flows) fairly well, but large discrepancies in the experimental data are observed for more complicated flow geometries or timedependent flows. In particular, significant gaps exist in the understanding of the behaviour of particle suspensions in open-channel flow.

In this study, we investigate the motion of suspensions consisting of neutrally buoyant non-colloidal particles. The flow geometry is the dam-break problem, that is, the flow of a fixed volume of suspension released suddenly down a slope. The goal is to gain new insight into the dynamics of time-dependent flows, especially the coupling between particle migration and bulk dynamics. The work is essentially experimental, with a major contribution being the simultaneous measurement of local flow properties (velocity and concentration) and macroscopic flow features (front position, depth evolution) for time-dependent flows with varying free boundaries. In contrast to earlier investigations, we took advantage of the visualization techniques that we specifically developed to measure velocity profiles inside the moving bulk (Wiederseiner et al. 2011; Andreini, Epely-Chauvin \& Ancey 2012).

In recent years, various authors have conducted dam-break or fully developed flow experiments to gain insight into the rheological behaviour of concentrated suspensions. Nsom (2000) ran dam-break experiments with buoyant particle suspensions (acrylic plastic beads immersed in glucose solutions) with solid concentration as high as $60 \%$. $\mathrm{He}$ found that, for solid concentrations lower than $52 \%$, the flow features (flow depth profile and position of the front with time) looked like those of a viscous flow, but for higher concentrations (typically $60 \%$ ), he observed that the flow came to a halt, a phenomenon typical of plastic behaviour. Timberlake \& Morris (2005) measured the velocity and concentration profiles in flows of thin films of concentrated suspensions down an inclined plane for various inclinations and concentrations. The data were successfully compared with the predictions of a particle-migration model. They also observed that the free surface became more deformed with increasing concentration or inclination. Bonnoit et al. (2010) used $40 \mu \mathrm{m}$ polystyrene bead suspensions in silicone oils in the $35-61 \%$ solids concentration range. By using the steady-state equation that relates the bulk viscosity to the surface velocity and flow depth, they were able to measure the viscosity for various flow depths and plane inclinations. They found that the bulk viscosity varied consistently with Zarraga's relation (Zarraga, Hill \& Leighton 2000). Non-buoyant particle suspensions have also been used in flume experiments. For instance, Ward et al. (2009) carried out experiments with particle concentrations ranging from 35 to $55 \%$. Various input parameters (particle density and size, fluid viscosity, initial volume, plane inclination) were varied. Among other variables, they measured the evolution of the front position $x_{f}(t)$. They showed that, even though departures from the $x_{f} \propto t^{1 / 3}$ theoretical trend were observed, this scaling was the correct approximation at long times.

We start the paper by outlining existing theories and addressing how they can be used to elucidate the influence of particle migration on the dynamics of suspension flows (see §2). While the dam-break problem for homogeneous Newtonian fluids is well known, the coupling with particle migration is more difficult. Even addressed 


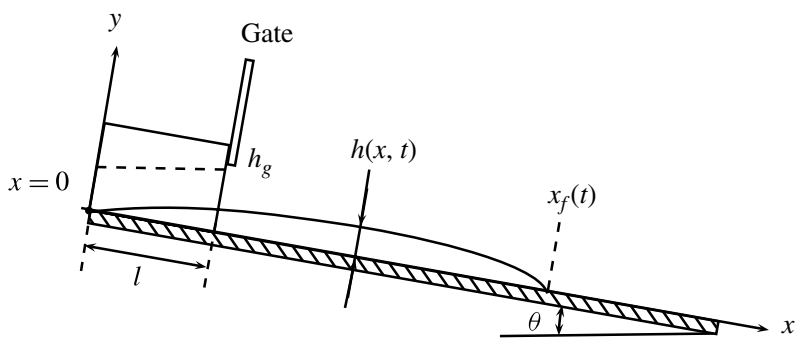

FIGURE 1. Schematic defining the flow configuration.

numerically, the fully coupled problem presents considerable difficulties, and we will touch upon a few aspects of this issue. We will then continue with a careful description of the experimental protocol (\$3). In $\S 4$, we present our experimental results, and in $\S 5$, we interpret them in light of particle migration theory.

\section{Theoretical perspective}

\subsection{Problem and notation}

We focus on the spread of a finite volume of particle suspension down an inclined flume (see figure 1). The suspension is composed of non-colloidal particles within a Newtonian carrier fluid of viscosity $\mu_{f}$ and surface tension $\sigma$. Both solid and fluid phases have the same density $\rho_{f}=\rho_{s}=\rho$. The particle concentration is denoted by $\phi$. The particles are assumed to be spheres of radius $a$. Initially a volume (per unit width) $A$ of suspension is contained in a reservoir. At time $t=0$, the material is released down a flume, which is tilted at an angle $\theta$ to the horizontal.

In what follows, we work mostly with non-dimensional flow variables, using hats to distinguish those variables that are dimensional. It is convenient to introduce such non-dimensional variables using scalings that reflect the shallowness of the flow:

$$
\hat{x}=L_{*} x, \quad \hat{y}=H_{*} y, \quad(\hat{u}, \hat{v})=U_{*}(u, \epsilon v), \quad \hat{t}=T_{*} t,
$$

where $H_{*}$ denotes the flow-depth scale, $T_{*}=L_{*} / U_{*}$ is the characteristic time, while $U_{*}=\rho g H_{*}^{2} \sin \theta / \mu$ and $L_{*}$ are the velocity and length scales, respectively, with $g$ the gravitational acceleration and $\mu$ the bulk viscosity, and $\epsilon=H_{*} / L_{*}$ is the aspect ratio. Owing to the possibility that the viscosity may be a flow variable, keep in mind that, in this scaling, $\mu$ refers to the bulk viscosity of a homogeneous suspension. If we use a relation such as the Krieger-Dougherty equation (2.2), we should define $\mu$ as $\mu=\mu(\bar{\phi})$, where $\bar{\phi}$ denotes the mean particle concentration. In (2.1) and figure 1 , we have defined a two-dimensional Cartesian coordinate system in which the $x$-axis points down the flume, the $y$-axis is in the direction of the upward-pointing normal, and the $z$-axis is the cross-stream direction. The rear end of the reservoir is chosen to be the origin of the $x$-axis. The bulk velocity $\boldsymbol{u}$ has components $u$ and $v$ in each of these directions; motion in the $z$-direction is discarded. To be consistent with volume conservation, we select the length and depth scales $L_{*}$ and $H_{*}$ such that $L_{*} H_{*}=A$, $L_{*}$ being the flume length. Because experimentally we will use a flume of finite width $W$, we also introduce the depth-to-width ratio: $\epsilon_{w}=H_{*} / W$. As emphasis is given to shallow gravity-driven flows of highly viscous fluids on slopes, flows are assumed to be in the limit of high capillary number and low Reynolds and aspectratio numbers: $C a=\mu U_{*} / \sigma \gg 1, \operatorname{Re}=\rho U_{*} H_{*} / \mu \ll 1$ and $\epsilon \ll 1$. The flow depth is 
denoted by $h(x, t)$. The front is the point at which the depth drops to zero. Its position is denoted by $x_{f}(t)$.

In the following, we are interested in the calculation of the front position $x_{f}(t)$, the depth profile $h(x, t)$ and the (streamwise) velocity and concentration fields $u(x, y, t)$ and $\phi(x, y, t)$. We will start with a calculation of the bulk flow properties by assuming that the suspension behaves as a homogeneous Newtonian fluid (\$2.2). Next we will investigate how particle migration alters the bulk dynamics (\$2.3). Here emphasis will be given given to the shear-induced diffusion model ( $\$ 2.4)$. There is another approach in common use: the suspension balance model, which is outlined in the supplementary material (available online at http://dx.doi.org/10.1017/jfm.2013.154), which also includes movies, further information on the experimental protocol and mathematical derivations.

\subsection{Motion of the bulk as a Newtonian fluid}

The simplest approach to the problem outlined above is to assume that the suspension is a Newtonian homogeneous fluid, whose bulk viscosity is given by an empirical equation such as the Krieger-Dougherty relation (Krieger \& Dougherty 1959)

$$
\eta(\phi)=\frac{\mu(\phi)}{\mu_{f}}=\left(1-\frac{\phi}{\phi_{m}}\right)^{-\beta},
$$

with $\phi_{m}$ the maximum particle concentration and $\beta$ a constant exponent $\left(\beta=5 \phi_{m} / 2\right.$ or $\beta=2$ are common values). Other expressions have been proposed for the viscosity function, in particular to better describe the viscosity behaviour in a wide range of flow conditions (Morris \& Boulay 1999; Zarraga et al. 2000; Boyer, Guazzelli \& Pouliquen 2011).

Using lubrication theory (Huppert 1982a; Ancey, Cochard \& Andreini 2009), we find that the shear stress distribution is given by

$$
\tau=(h-y)\left(1-\epsilon \cot \theta \frac{\partial h}{\partial x}\right),
$$

from which we infer that the velocity profile takes a parabolic shape, whose amplitude is modulated by the free surface gradient

$$
u(x, y, t)=\frac{1}{2} y(2 h-y)\left(1-\epsilon \cot \theta \frac{\partial h}{\partial x}\right) .
$$

Conservation of mass leads to the governing equation for the flow depth $h(x, t)$, which is a nonlinear advection-diffusion equation

$$
\frac{\partial h}{\partial t}+\frac{1}{3} \frac{\partial h^{3}}{\partial x}=\frac{1}{3} \epsilon \cot \theta \frac{\partial}{\partial x}\left(h^{3} \frac{\partial h}{\partial x}\right) .
$$

The only exact solutions available are for horizontal bottoms $(\theta=0)$ and an infinitely wide flume $\left(\epsilon_{w} \rightarrow 0\right)$, for which the governing equation reduces to a nonlinear diffusion equation (Huppert 1982b). For other cases, asymptotic solutions can be obtained at short and long times (Huppert 1982a; Ancey et al. 2009). For instance, it can be shown that, at sufficiently long times, the front position of a fluid avalanche over a tilted plane can be approximated by

$$
x_{f}=x_{f 0}+\epsilon x_{f 1}+\cdots=\left(\frac{9}{4} t\right)^{1 / 3}+\epsilon \cot \theta\left(\ln 2-\frac{1}{2}\right)\left(\frac{3}{2 t}\right)^{1 / 3}+O\left(\epsilon^{2}\right) .
$$


This asymptotic expansion is obtained by seeking similarity solutions to (2.5) and using matched asymptotic expansions (Ancey et al. 2009; Andreini et al. 2012). The zeroth-order term on the right-hand side of (2.6) represents the advection of the flowing mass, while the first-order correction accounts for diffusive effects within the leading edge (influence of the free-surface gradient). To leading order, the flow depth is the composite of the inner and outer solutions (Andreini et al. 2012)

$$
h(x, t)=\sqrt{\frac{x}{t}}+\sqrt{\frac{x_{f 0}}{t}}\left\{K^{-1}\left[\frac{x-x_{f 0}}{\epsilon \cot \theta}\left(\frac{3}{2 t}\right)^{-2 / 3}\right]-1\right\},
$$

where $K(h)=h-\operatorname{arctanh} h+\ln 2-\frac{1}{2}$. In these asymptotic solutions, the expansions involve the aspect ratio $\epsilon$. First-order and higher-order contributions represent the perturbations induced by the gradient of the free surface, which is especially significant within the tip region near the front. Another perturbation to the advection of the flowing mass is caused by sidewall friction. In the supplementary material, we derive an approximate solution that takes this effect into account. In that case, the expansions involve the depth-to-width ratio $\epsilon_{w}$. We have found that the front position is approximated by

$$
x_{f}(t)=\left(\frac{9}{4} t\right)^{1 / 3}-2 \epsilon_{w}-\epsilon_{w}^{2}\left[\frac{35}{3}\left(\frac{2}{3 t^{2}}\right)^{1 / 3}+2\left(\frac{12}{t^{2}}\right)^{1 / 3}\right]+O\left(\epsilon_{w}^{3}\right),
$$

while the depth profile is given by

$$
h(x, t)=\sqrt{\frac{x}{t}}+\epsilon_{w} \frac{8}{3} \frac{x}{t}+\epsilon_{w}^{2} \frac{70}{9}\left(\frac{x}{t}\right)^{3 / 2}+\cdots .
$$

\subsection{Particle migration}

In particle suspensions, particle migration is expected to play a significant role (Morris 2009). Particle migration induces a concentration stratification across the stream, which leads to a local change in the viscosity and thus velocity profile. In polydisperse systems, particle size segregation (grain sorting) may also contribute to increasing concentration inhomogeneity (Chang \& Powell 1994; Krishnan, Beimfohr \& Leighton 1996; Lyon \& Leal 1998b), but we will leave this problem aside for the moment. Note also that particles can migrate laterally in the $z$-direction in open-channel flows, but assuming that $\epsilon_{w} \ll 1$, we will neglect the particle flux in this direction.

Among the numerous models proposed for describing particle migration, two approaches have attracted more attention over the years. The first class of models takes its root in the phenomenological description of particle migration in Couette flows (Leighton \& Acrivos 1987). Particles migrate mainly as a result of a shearrate gradient. The particle flux is directed towards the zone of lower shear rate: $\boldsymbol{j} \propto-\phi^{2} \nabla \dot{\gamma}$, where $\boldsymbol{j}=\phi\left(\boldsymbol{u}^{p}-\boldsymbol{u}\right)$ is the particle flux relative to the suspension velocity $\boldsymbol{u}$, and $\boldsymbol{u}^{p}$ is the particle phase velocity. In the other class, migration is the response to an imbalance in the particle stress distribution: $\boldsymbol{j} \propto-\nabla \cdot \boldsymbol{\Sigma}^{p}$. This relation was derived from the microstructural analysis of the particle and fluid phase contributions to the stress tensor (Nott \& Brady 1994; Morris \& Boulay 1999). The theoretical underpinning is still actively debated (Lhuillier 2009; Nott, Guazzelli \& Pouliquen 2011). 


\subsection{Shear-induced migration model}

Phillips et al. (1992) developed a phenomenological model that describes migration as a diffusive process that combines two contributions:

$$
j=-\phi K_{c} \frac{\epsilon_{a}^{2}}{\epsilon} \nabla(\phi \dot{\gamma})-K_{\mu} \dot{\gamma} \phi^{2} \frac{\epsilon_{a}^{2}}{\epsilon} \frac{\mathrm{d} \ln \eta}{\mathrm{d} \phi} \nabla \phi,
$$

where $\epsilon_{a}=a / H^{*}$ is the typical ratio of the particle radius to the flow depth. Note that the scale for the particle flux is $J_{*}=\epsilon U_{*}$, as the particle flux is directed upwards. The first contribution on the right-hand side of (2.10) is a shear-induced diffusion flux similar to the original phenomenological form of Leighton \& Acrivos (1987); this particle flux reflects the anisotropy in the two-particle encounter rate. The second contribution has the form of nonlinear Fickian diffusion and accounts for the displacements induced by two-particle encounters. Phillips et al. (1992) introduced two constant parameters $\left(K_{c}=0.43\right.$ and $\left.K_{\mu}=0.65\right)$, while Lyon \& Leal (1998a) showed that the best agreement was obtained with $K_{c} / K_{\mu}=0.77$. Further experimental investigations support a dependence of $K_{\mu}$ and $K_{c}$ on $\phi$. For instance, Tetlow et al. (1998) found that $K_{c} / K_{\mu}=1.042 \phi+0.1142$. The diffusive flux model leads to an evolution equation for the particle concentration in the form of an advection-diffusion equation coupled to the momentum balance equations

$$
\frac{\partial \phi}{\partial t}+u \frac{\partial \phi}{\partial x}+v \frac{\partial \phi}{\partial y}=-\nabla \cdot j .
$$

Coupling this evolution equation to our time-dependent flow problem leads to substantial difficulties, including shock formation and loss of hyperbolicity (Cook, Bertozzi \& Hosoi 2008; Gray \& Ancey 2009), whose solution goes far beyond the scope of the present paper. Physical insights can be gained by looking at special forms of (2.11).

Of particular interest are the forms of the fully developed velocity and concentration profiles. The steady-state solution corresponds to the situation in which the particle flux $\boldsymbol{j}$ vanishes. Adopting the Krieger-Dougherty viscosity function (2.2) and integrating the differential equation (2.10) leads to the concentration profile in an implicit form

$$
\frac{\phi_{w}}{\phi}\left(\frac{\phi_{m}-\phi}{\phi_{m}-\phi_{w}}\right)^{\beta(\alpha-1)}=1-\frac{y}{h},
$$

with $\alpha=K_{\mu} / K_{c}$ and where $\phi_{w}$ is the particle concentration at the wall. As we expect that the particle concentration increases when approaching the free surface, the ratio $\alpha$ should satisfy $\alpha \geqslant 1$. In that case, for all concentrations, the model predicts that the particle concentration increases from $\phi_{w}$ at the bottom to $\phi_{m}$ at the free surface. For particular values of $\beta$ and $\alpha$, we can simplify this implicit equation. For $\beta=2$ and $\alpha=3 / 2$, which are values reasonably close to the ones found experimentally by Phillips et al. (1992), we get

$$
\phi=\frac{\phi_{w} h}{\phi_{m} h-\left(\phi_{m}-\phi_{w}\right) y} \quad \text { with } \frac{\phi_{w}}{\phi_{m}-\phi_{w}} \log \frac{\phi_{m}}{\phi_{w}}=\bar{\phi},
$$



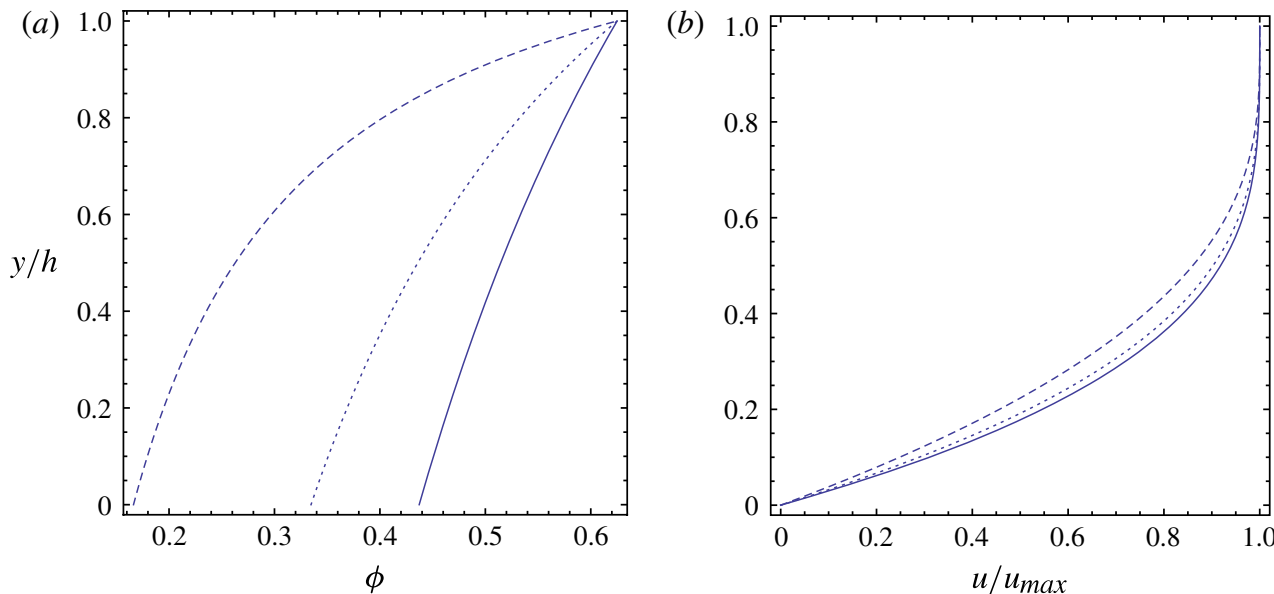

FIGURE 2. (Colour online) (a) Particle concentration and $(b)$ velocity profiles predicted by (2.13) for three mean concentrations $\bar{\phi}=30 \%$ (dashed), $\bar{\phi}=45 \%$ (dotted) and $\bar{\phi}=52 \%$ (solid). The Krieger-Dougherty relation has been used with $\beta=2$. For the migration law proposed by Phillips et al. (1992), we have taken $\alpha=3 / 2$.

where $\bar{\phi}$ is the mean concentration of the suspension. When substituted into the momentum balance equation

$$
\dot{\gamma}=\frac{\bar{\eta}}{\eta(\phi)}(h-y),
$$

where $\bar{\eta}$ is a shorthand notation for $\eta(\bar{\phi})$, this relation leads to an analytical expression for the velocity (which we do not report here due to its complexity). The model shows that the higher the concentration, the blunter the velocity profile. Figure 2 provides a typical example for three mean concentrations $\bar{\phi}=30,45$ and $52 \%$. It is interesting to quantify the degree of blunting of the velocity profile. To that end, we fit a power-law function to the numerical velocity profiles

$$
u=\kappa\left(h^{n}-(h-y)^{n}\right),
$$

with $\kappa$ a fitting parameter and $n$ an index that quantifies the blunting. This equation has no physical meaning (for example, it does not mean that the rheological behaviour is shear-thinning). In the absence of blunting, the suspension behaves like a Newtonian fluid and the velocity profile takes a parabolic shape, thus $n=2$. As the particles migrate, the velocity profile becomes blunter. The larger the value of $n$, the blunter the velocity profile. We also introduce a non-parametric blunting index, i.e. an index that does not depend on a particular parametrization of the velocity profile,

$$
m=\frac{\int_{0}^{h} u(y, t) \mathrm{d} y}{h u(h, t)}=\frac{\bar{u}}{u(h, t)},
$$

which is simply the ratio of the mean velocity $\bar{u}$ to the surface velocity $u(h, t)$. This ratio takes the value $2 / 3$ for a (Newtonian-like) parabolic profile and 1 for a perfectly uniform (blunted) profile. If the actual velocity profile conforms to a power law, then the relation $m=n /(1+n)$ holds. 


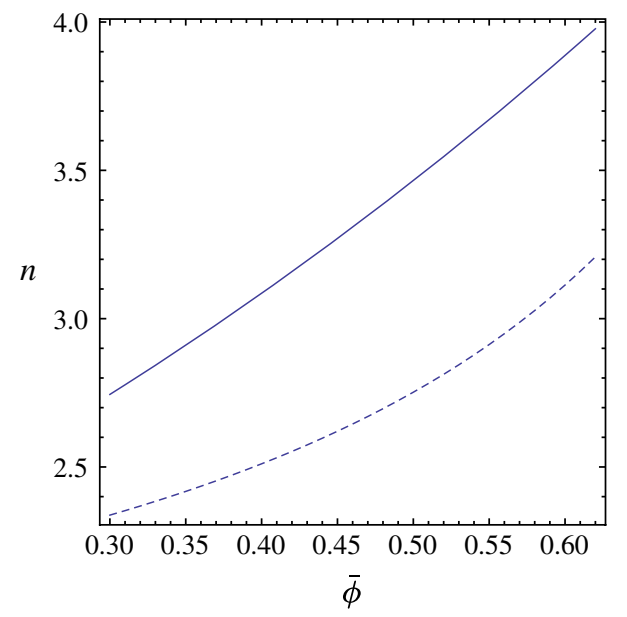

FIGURE 3. (Colour online) Variation in the blunting index $n$ as a function of the mean particle concentration. The $n$ values were determined by fitting a power-law function to the velocity profile using the shear-induced diffusion model and (2.12) with $\beta=2$ and $\alpha=3 / 2$ (solid) or $\beta=2$ and $\alpha=1.042 \phi+0.1142$ (dashed).

Figure 3(a) shows how $n$ varies with the mean particle concentration depending on the values of the parameters $\alpha$ and $\beta$. Small changes in these values result in large changes in the value of $n$, which shows the great sensitivity of the model to its parameters.

Let us now consider the case of uniform film flows where the concentration gradient in the flow direction is zero $\left(\partial_{x} \phi=0\right)$. The normal velocity $v$ is zero while the shear rate is given by the momentum balance equation (2.14). The governing equation for the particle concentration reduces to a nonlinear parabolic equation,

$$
\frac{\partial \phi}{\partial t}=K_{c} \bar{\eta} \frac{\epsilon_{a}^{2}}{\epsilon} \frac{\partial}{\partial y}\left(\phi \frac{\partial}{\partial y}\left(\frac{\phi}{\eta(\phi)}(h-y)\right)+\alpha \frac{\phi^{2}}{\eta(\phi)}(h-y) \frac{\mathrm{d} \ln \eta}{\mathrm{d} \phi} \frac{\partial \phi}{\partial y}\right) .
$$

We then find that the time scale for reaching a steady state is approximately

$$
t_{c} \sim \frac{\epsilon}{\epsilon_{a}^{2}}=\frac{H_{*}^{3}}{a^{2} L_{*}}
$$

a scaling that is close to the relation obtained by Nott \& Brady (1994) and Norman, Nayak \& Bonnecaze (2005) except for the weighting coefficient (Nott \& Brady (1994) established that $\hat{t}_{s s} \approx H_{*}^{3} /\left(a^{2} \bar{u}\right)$ in its dimensional form). We solved the evolution equation (2.17) numerically by assuming a no-flux condition at the boundaries $(\boldsymbol{j}=0$ at $y=0$ and $y=h)$ and a uniform profile as the initial condition $(\phi=\bar{\phi}$ at $t=0)$. The convergence time $t_{s s}$ was defined numerically as the time taken until which the relative difference between the time-dependent and steady-state solutions was less than $1 \%$. Figure 4 shows how this time varies with the mean solid fraction $\bar{\phi}$. The simulations were conducted for different values of the ratio $\epsilon / \epsilon_{a}^{2}$ and $\bar{\phi}$. We found that, for sufficiently high particle concentrations $\left(\phi / \phi_{m}>0.6\right)$, the convergence time was 


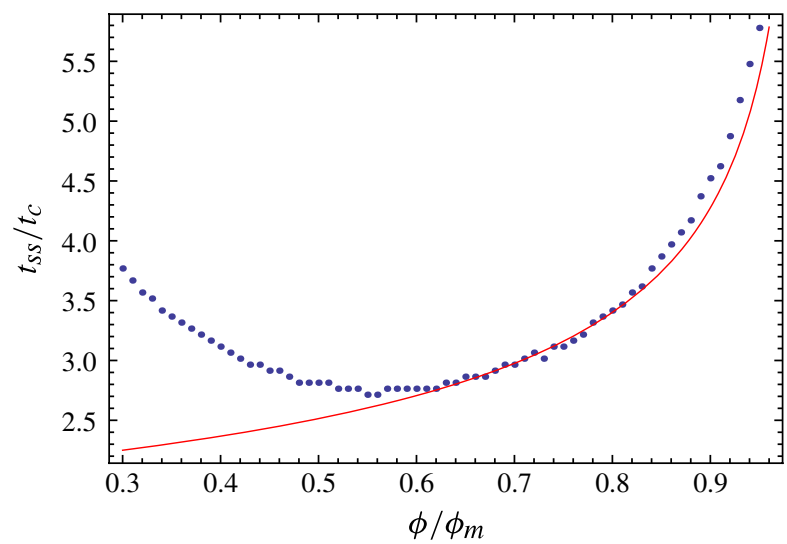

FIgURE 4. (Colour online) Convergence time as a function of $\bar{\phi}$ (points). Also plotted is the empirical trend $t_{s s}=2 t_{c}\left(1-\phi / \phi_{m}\right)^{-1 / 3}$ (solid). The Krieger-Dougherty relation has been used with $\beta=2$. We have used the migration law proposed by Phillips et al. (1992) with $\alpha=3 / 2$.

approximately

$$
t_{s s}=2 t_{c}\left(1-\frac{\phi}{\phi_{m}}\right)^{-1 / 3},
$$

which shows that the time taken to reach steady state is influenced by the flow geometry (through the ratios $\epsilon_{a}^{2}$ and $\epsilon$ ) rather than the solids fraction, except in the close vicinity of the maximum concentration. The exponent in (2.19) depends strongly on the value of $\alpha$; for instance, if we take $\alpha=(1.042 \phi+0.1142)^{-1}$ as suggested by Tetlow et al. (1998) instead of $\alpha=3 / 2$, we found that $t_{s s} \approx 2 t_{c}\left(1-\phi / \phi_{m}\right)^{-3 / 5}$. Note also that, strictly speaking, (2.19) pertains to the concentration profiles, but it is also relevant for the velocity profiles, as the local velocity is dictated by the local concentration through (2.14).

Figure 5(a) shows the evolution of the concentration profile from initial to steady states for $\bar{\phi}=52 \%$. We notice that the concentration at the free surface jumps instantaneously to the maximum solids fraction (due to the non-flux condition imposed at the free surface), whereas the bottom concentration decreases continuously to the steady-state value. Using the momentum balance equation (2.14), we computed the streamwise velocity component. Figure $5(b)$ shows the evolution of the velocity profile. Initially, as the concentration was uniform, the velocity profile was parabolic, but with time, it became more blunt near the free surface. As the concentration decreased at the base of the flow, wherein shear was concentrated, the velocities increased with time. The numerical solution to (2.17) showed that the free-surface velocity increased by a factor of more than 2. From this standpoint, particle migration should result in a noticeable increase of flow velocity compared to a homogeneous suspension. To characterize the degree of blunting, we fitted a power-law function (2.15) to the numerical data. Figure 5(c) shows how the exponent $n$ varies with time. At short times, as the velocity profile was initially parabolic, the $n$ value was close to unity. It then decreased continuously with time until it reached its steady-state value for $t \geqslant t_{s s}$. We also found that, in the transition regime, the growth was fairly well captured by a 


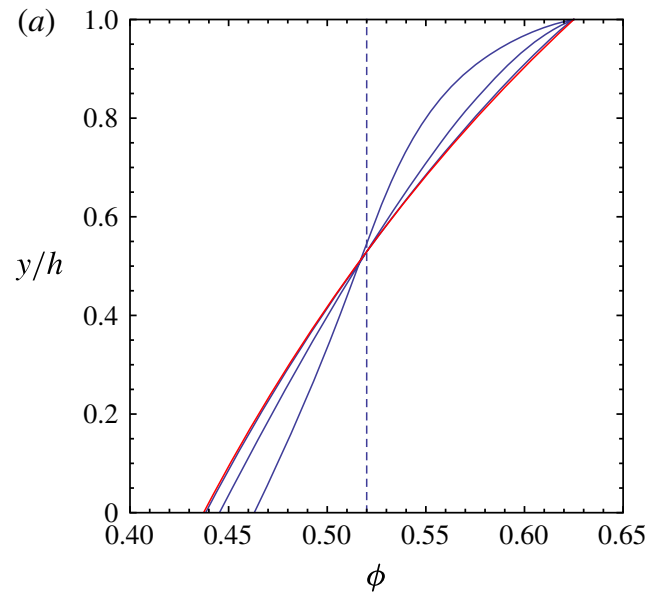

(b)

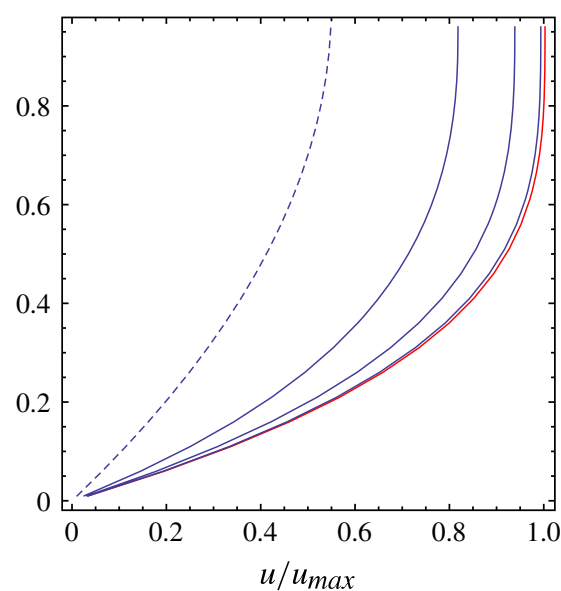

(c)

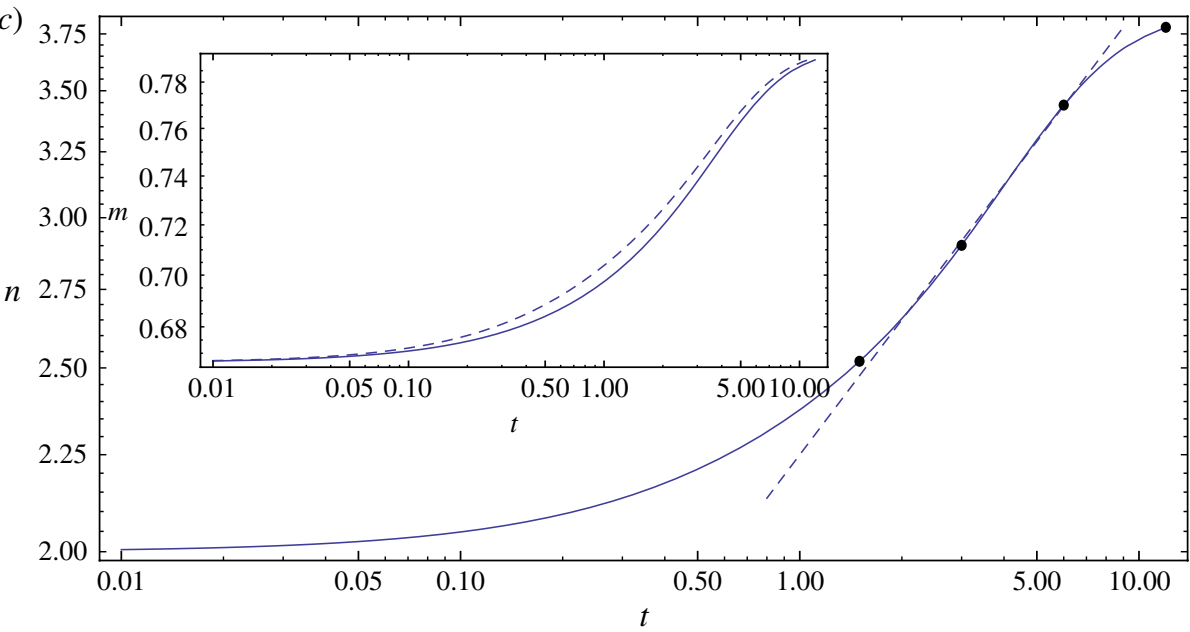

FIGURE 5. (Colour online) Evolution of the $(a)$ concentration and $(b)$ velocity profiles at (in order) times $t=0$ (dashed), 1.5, 3, 6 (solid) and 12 (red online). The velocity has been scaled by the maximum velocity (at the free surface) reached by the steady-state solution: $u_{\max }=\left[\phi_{m}-5 \phi_{w}+2 \phi_{w}\left(3 \bar{\phi}-\phi_{w}\right)\right] /\left[2\left(\phi_{m}-\phi_{w}\right)\right]$. (c) Evolution of the degree of blunting $n$ with time; the dashed line represents the trend $n=2.2 t^{1 / 4}$; the dots stand for the times at which the velocity and concentration profiles were computed. Inset: plot of the evolution of the non-parametric blunting index $m$ (solid). On the same plot, we have also plotted the curve $n /(n+1)$ (dashed) where $n$ is the blunting index shown in the main panel. Numerical computations were done with $\bar{\phi}=52 \%, K_{c} \bar{\eta} \epsilon_{a}^{2} / \epsilon=1, \beta=2$ and $\alpha=3 / 2$. The time to convergence is $t_{s s}=3.56$.

power-law function

$$
n=2.2 t^{1 / 4} \text {. }
$$

While the steady-state value was strongly dependent on the parameters $\alpha$ and $\beta$, the transition regime, in particular the growth rate, was weakly dependent on $\alpha$ and $\beta$, but markedly dependent on $\bar{\phi}$. To test the performance of the power-law model (2.15) used to describe the velocity profiles, we have plotted the non-parametric index (2.16) in the 


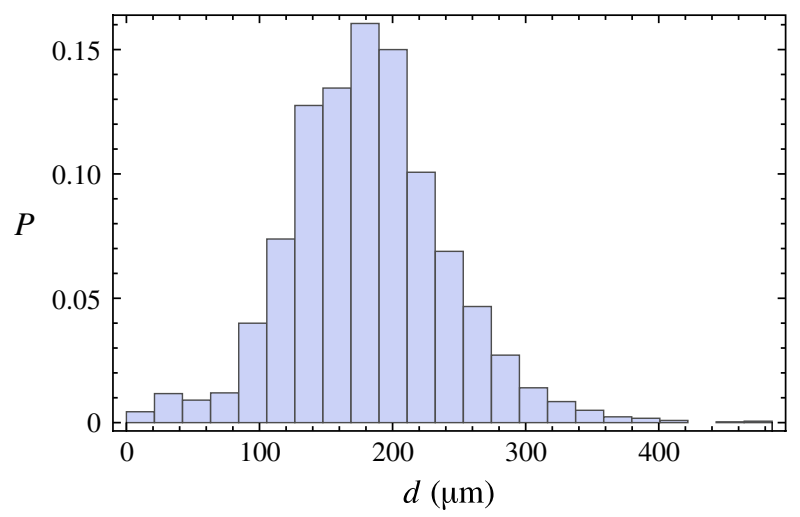

FIGURE 6. (Colour online) Grain size distribution of PMMA particles used in our experiments. The histogram was obtained by measuring the particle diameter using an epifluorescent microscope (Olympus BX60). The sample size was 3430 particles.

inset of figure $5(c)$ together with the ratio $n /(n+1)$. The two curves being quite close to each other, we conclude that the power-law model is a fairly good approximation of the velocity profiles.

\section{Experimental facility and procedure}

\subsection{Suspension composition}

All experiments (except for one) were run with concentrated suspensions of poly(methyl methacrylate) (PMMA) particles within a mixture with three components, called trimix. We adapted the fluid mixture composition originally described by Lyon \& Leal (1998a). The particle density and refractive index were matched by a fluid mixture with mass fractions of $50 \%$ Triton X100, $28 \%$ 1,6-dibromohexane (DBH) and $22 \%$ UCON oil (75-H450 oil from Dow Chemicals). The density mismatch between the fluid and solid phases was zero to within $5 \times 10^{-4} \mathrm{~g} \mathrm{~cm}^{-3}$. The particles and fluids had the same refractive index to within $10^{-4}$. The final mixture density was $\rho=1.184 \pm 0.0005 \mathrm{~g} \mathrm{~cm}^{-3}$, the refractive index was measured using an Atago rx5000a refractometer at $532 \mathrm{~nm}$ and $20^{\circ} \mathrm{C}$ : we found $n_{r}=1.4885 \pm 0.0002$. The viscosity of the interstitial fluid was measured using a Bohlin CVOR rheometer equipped with a cone-and-plate geometry: we found $\mu_{f}=0.124 \pm 0.003 \mathrm{~Pa} \mathrm{~s}$ at $20^{\circ} \mathrm{C}$. Its surface tension was measured using the pendant drop method (Hansen \& Rødsrud 1991): we found $\sigma=33 \pm 5 \mathrm{mN} \mathrm{m}^{-1}$. The uncertainty concerning the bulk density resulted from various processes (e.g. errors in the temperature control, errors in the fluid-density measurements). We used PMMA particles, manufactured by Altuglass (la GarenneColombes, France). Figure 6 shows that the particle size distribution was bell-shaped, with a mean particle diameter of $190 \mu \mathrm{m}$ and a standard deviation of $60 \mu \mathrm{m}$. Preparing a transparent suspension of neutrally buoyant particles is a complicated process, which requires considerable attention at every step. See the supplementary material for the details.

\subsection{Flume}

Experiments were conducted in a flume with aluminium sidewalls. The flume bottom was made from the same material (PMMA) as the particles, which made it possible to create a vertical laser sheet passing through the bottom, whilst keeping the refraction 


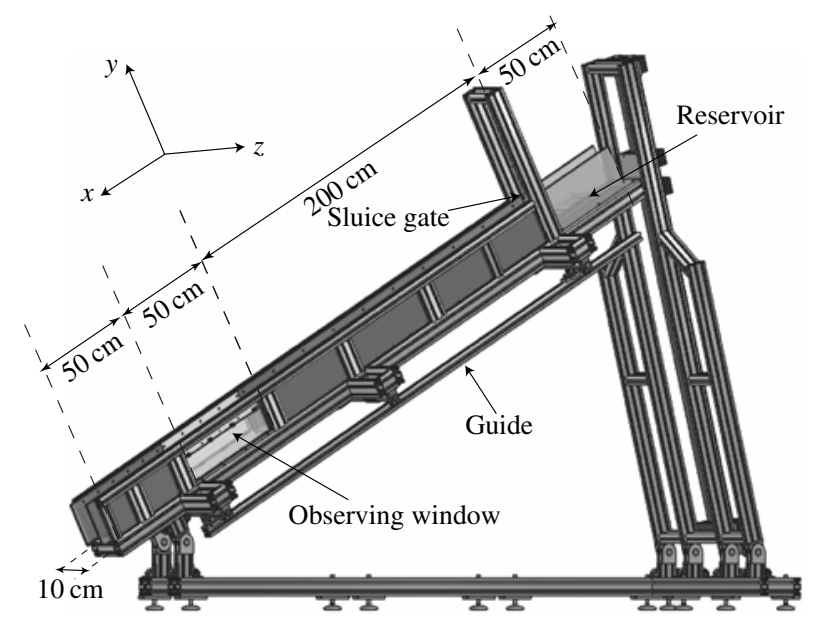

FIgURE 7. Flume used for the experiments. For this flume, we define a two-dimensional Cartesian coordinate system similar to the one used in figure 1: the $x$-axis points down the flume, the $y$-axis is in the direction of the upward-pointing normal, and the $z$-axis is the cross-stream direction. The upper end of the flume is at $\hat{x}=0$, while the lower end is at $\hat{x}=350 \mathrm{~cm} ; \hat{z}=0$ refers to the right sidewall (when looking at the flume from the inlet), while $\hat{z}=W=10 \mathrm{~cm}$ refers to the left sidewall.

angle between the flow and the bottom constant, and taking images from below. Figure 7 shows a sketch of the facility. An optical glass pane, located $50 \mathrm{~cm}$ from the flume outlet, was inserted to observe the flows from the side. The flume was $3.5 \mathrm{~m}$ long and $10 \mathrm{~cm}$ wide. It could be inclined from 0 to $35^{\circ}$. Its position was accurately controlled using a digital inclinometer with a precision of $0.1^{\circ}$. The upper part of the flume was equipped with a sluice gate mounted on a pneumatic jack and was used as a reservoir. The gate was an ultrahigh-molecular-weight polyethylene plate, which could be removed in less than $0.5 \mathrm{~s}$. An electromagnetic sensor located on the jack gave the reference time for the experiment and synchronized the other instruments. In order to keep the temperature of the suspension constant during the experiment, the flume was enclosed in an isolated room, whose temperature was fixed at $20.0 \pm 0.2{ }^{\circ} \mathrm{C}$ using a fan coil unit.

\subsection{Measurement systems}

We measured different flow variables (e.g. front position, flow depth), but the emphasis was given to flow visualization. We measured the velocity profiles in the direction normal to the flume bottom and, with less success, particle concentration profiles using high-speed cameras and particle image velocimetry (PIV) techniques. For PIV measurements, we used a Basler A504k camera working at 200 to $1270 \mathrm{~Hz}$ (depending on front velocity), mounted with a Nikkor $105 \mathrm{~mm}$ macro lens with an aperture of $\mathrm{f} / 4$. The images were then processed using classic PIV techniques (Raffel et al. 2007). Velocity fields were computed using the open-source software called MatPIV (Sveen 2004). The particle concentration was estimated by counting the number of particles inside a given window, then taking the time average. The typical window size was $700 \mathrm{px} \times 20 \mathrm{px}$, i.e. $35 \mathrm{~mm} \times 1 \mathrm{~mm}$, and the time length over which integration was performed was 100 images, i.e. from 0.08 to $0.40 \mathrm{~s}$. Relative 


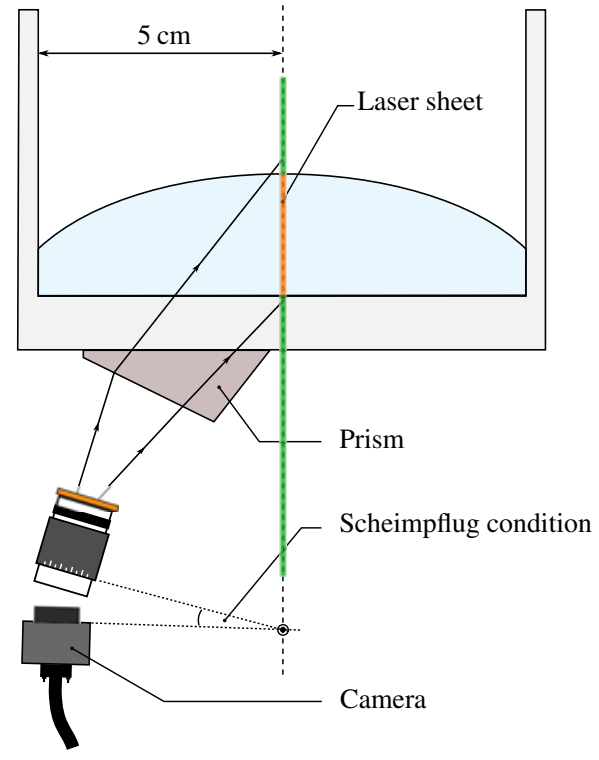

FIGURE 8. (Colour online) Sketch of the measurement system for the velocity profiles within the moving suspension. Because of the suspension-air interface and the three-dimensional nature of the flows, we were forced to film the flow from below. When taking images with a camera whose sensor (CCD) is not parallel to the object, one can use the Scheimpflug principle, which involves tilting the camera until the image plane (on the $\mathrm{CCD}$ ), the lens plane and the object plane (lit by the laser sheet) have a common line of intersection (see chap. 7 in Raffel et al. (2007) for additional information).

uncertainties on local $\phi$ measurements did not exceed $2 \%$ for $\hat{y} \leqslant 60$ px and $4 \%$ for $60 \leqslant \hat{y} \leqslant 80 \mathrm{px}$, but increased tremendously for $\hat{y}>100 \mathrm{px}$.

Figure 8 explains how we measured velocity profiles. Taking images from the side was possible with uniform flows, but much more restrictive from the optical standpoint for non-uniform flows. For instance, filming from the side did not allow measurement of velocities within the head far from the sidewall due to the strong curvature of the free surface. Moreover, as we used a fairly wide flume and measured profiles along the centreline $(5 \mathrm{~cm}$ from the sidewall), we needed to be able to see through $5 \mathrm{~cm}$ of suspension, i.e. approximately 1000 fluid-particle interfaces, which went beyond the current capacities (Wiederseiner et al. 2011). The idea was thus to film from below the flume bottom. Owing to the much shorter optical path, the images were of much better quality and were not altered by the curvature of the free surface. This technique had, however, the disadvantage of producing blurred and distorted images if no corrective action was taken. To get around this problem, we adjusted the inclinations of the camera charge-coupled device (CCD) and the lens so that the Scheimpflug rule was satisfied (the image was then in focus). A prism (made up of a PMMA block, with the same refractive index as that of the flume bottom) was also needed to avoid refraction. As this system caused significant image distortion, we had to correct it to compute the velocity field properly; this was done by taking an image of a test chart and using the Matlab built-in function $c p 2$ tform to undistort the images. 


$\begin{array}{lccccccccc}\text { Run } & \begin{array}{c}\bar{\phi} \\ (\%)\end{array} & \begin{array}{c}\text { Mass } \\ (\mathrm{g})\end{array} & \begin{array}{c}\rho_{p} \\ \left(\mathrm{~kg} \mathrm{~m}^{-3}\right)\end{array} & \begin{array}{c}\rho_{f} \\ \left(\mathrm{~kg} \mathrm{~m}^{-3}\right)\end{array} & \begin{array}{c}\mu_{f} \\ (\mathrm{mPa} \mathrm{s})\end{array} & \begin{array}{c}\theta \\ (\mathrm{deg} .)\end{array} & \begin{array}{c}T_{*} \\ (\mathrm{~s})\end{array} & \begin{array}{c}U_{*} \\ \left(\mathrm{~m} \mathrm{~s}^{-1}\right)\end{array} & R e \\ \mathrm{~A} & 30 & 3000 & 1184 & 1184 & 124 & 25 & 2.41 & 1.056 & 27 \\ \mathrm{~B} & 45 & 3000 & 1184 & 1184 & 124 & 25 & 8.32 & 0.306 & 2.3 \\ \mathrm{C} & 52 & 3000 & 1184 & 1184 & 124 & 5 & 112 & 0.022 & 0.06 \\ \mathrm{D} & 52 & 3000 & 1184 & 1184 & 124 & 15 & 37.7 & 0.067 & 0.18 \\ \mathrm{E} & 52 & 3000 & 1184 & 1184 & 124 & 25 & 23.1 & 0.110 & 0.29 \\ \mathrm{~F} & 55 & 3000 & 1184 & 1184 & 124 & 25 & 45.3 & 0.056 & 0.08 \\ \mathrm{G} & 56 & 3000 & 1184 & 1184 & 124 & 25 & 60.3 & 0.042 & 0.04 \\ \mathrm{H} & 57 & 3000 & 1184 & 1184 & 124 & 25 & 84.2 & 0.030 & 0.02 \\ \mathrm{I} & 52 & 6000 & 1184 & 1184 & 124 & 25 & 5.78 & 0.441 & 2.4 \\ \mathrm{~J} & 57.5 & 6000 & 1184 & 1184 & 124 & 25 & 25.49 & 0.100 & 0.12 \\ \mathrm{~K} & 52 & 3000 & 1184 & 1068 & 446 & 25 & 79.2 & 0.032 & 0.02\end{array}$

TABLE 1. Features of the different runs: particle concentration, initial mass, particle density, fluid density, fluid viscosity, flume inclination, time scale, velocity scale and Reynolds number. For all runs except run K, the interstitial fluid was trimix. For run K, we used a binary mixture (UCON oil and Triton). The scales were defined in $\S 2.1$. The Krieger-Dougherty equation (2.2) (with $\beta=2$ and $\phi_{m}=62.5 \%$ ) has been used to define the bulk viscosity $\mu(\bar{\phi})$.

\section{Experimental results}

\subsection{Overview}

We conducted several experimental campaigns to investigate how the flow dynamics of a fixed volume of suspension was influenced by the flume inclination, initial volume, particle concentration and fluid viscosity. Here we have selected 11 runs; see Andreini (2012) for further information. Table 1 summarizes the different runs presented in this paper. Recall that, in the following, all the flow variables are dimensionless variables, given by the scaling relation (2.1). Here we take $L_{*}=2.55 \mathrm{~m}$ as the length scale. Table 1 reports the time scale $T_{*}$, the velocity scale $U_{*}$ and the resulting flow Reynolds number $R e=\rho U_{*} H_{*} / \mu(\bar{\phi})$. Other scales depend on particular flow conditions and can be calculated by referring to table 1 . Note that the run duration was constrained by the acquisition rate of our cameras (in the $250-1270 \mathrm{~Hz}$ range for the runs presented here) and the memory size, as the images were stored in the PC memory before being written to the hard drive (the typical sequence size was $80 \mathrm{~GB}$ ).

Except for run $\mathrm{K}$, all suspensions contained neutrally buoyant particles as described in $\S 3$. For run $\mathrm{K}$, there was slight negative buoyancy $\left(\Delta \rho=116 \mathrm{~kg} \mathrm{~m}^{-3}\right.$, i.e. $10 \%$ of particle density), which caused slow sedimentation. For particle concentrations in excess of $57.5 \%$, we observed a complicated behaviour involving a macro-viscous regime at short times, then a stick-slip regime at longer times. This flow behaviour is described in two other papers (Ancey, Andreini \& Epely-Chauvin 2013; Andreini, Ancey \& Epely-Chauvin 2013).

\subsection{Front position}

Among the macroscopic flow features, the front position is of paramount importance, not only because it was easy to track, but also because the dependence of $x_{f}$ on $t$ yields insight into the rheological behaviour. Figure 9 shows the front position as a function of time in a dimensionless $\log -\log$ form for all runs. In addition to the experimental data, we have also plotted the similarity solution $x_{f}=(9 t / 4)^{1 / 3}$ and the asymptotic expansions (2.6) and (2.8) up to order two. On the whole, the $x_{f} \propto t^{1 / 3}$ 


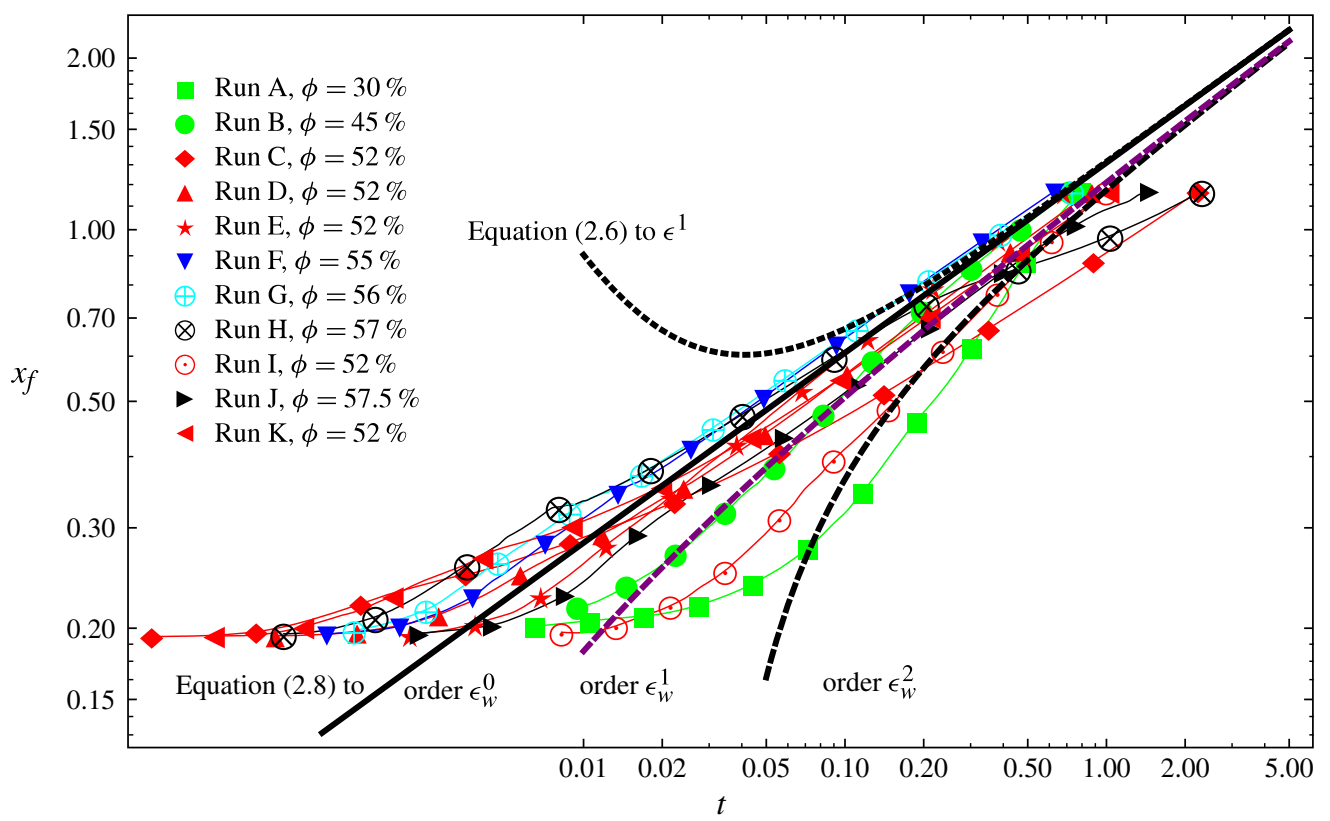

FIgure 9. (Colour online) Position of the front as a function of time. We also report the theoretical approximations: the similarity solution (2.6) or (2.8) to order zero (solid), the firstorder correction when diffusive effects due to the pressure gradient are taken into account, (2.6) up to order one (dotted), and the first- and second-order corrections (dashed) when sidewall friction is taken into account in (2.8). The first- and second-order corrections were computed using the typical values (for our runs) $\epsilon_{w}=0.01, \epsilon=0.004$ and $\theta=25^{\circ}$.

scaling offers a proper description of the front evolution, which justifies that we refer to these flows as macro-viscous. Some departures from the power-law scaling are seen. For $\phi=55$ and $56 \%$, the experimental curves lie slightly above the similarity solution, which may be an indication of diffusive effects. For low concentrations $(\phi=30$ and $45 \%$ ), the experimental curves are below the similarity solution and their variations are grossly captured by the sidewall correction terms. The largest difference between the similarity solution and experimental data is observed for the shallowest slopes (runs $\mathrm{C}$ and $\mathrm{D}$, respectively, $\theta=5$ and $15^{\circ}$ ) or the highest concentrations (runs $\mathrm{H}$ and J, respectively, $\phi=57$ and $57.5 \%$ ). The deviation observed for shallow slopes could have been anticipated, as it was also observed with genuinely Newtonian fluids in a similar flow configuration (Andreini et al. 2012). In contrast, we observed that, for the highest concentrations, the experimental curves diverged from the similarity solution at long times, and this departure was even more marked when increasing the particle concentration to 58 or $59 \%$ (the corresponding data are not shown here).

\subsection{Flow depth and velocity profiles}

From our observing window located at $\hat{x}=255 \mathrm{~cm}(x=1$ in dimensionless form) from the flume inlet, we measured the evolution of the depth profile, and, by taking images from below, we determined the velocity profiles at different times. To illustrate the influence of the particle concentration on the velocity profiles, we plotted the experimental data corresponding to the same flume inclination $\left(\theta=25^{\circ}\right)$, the same 

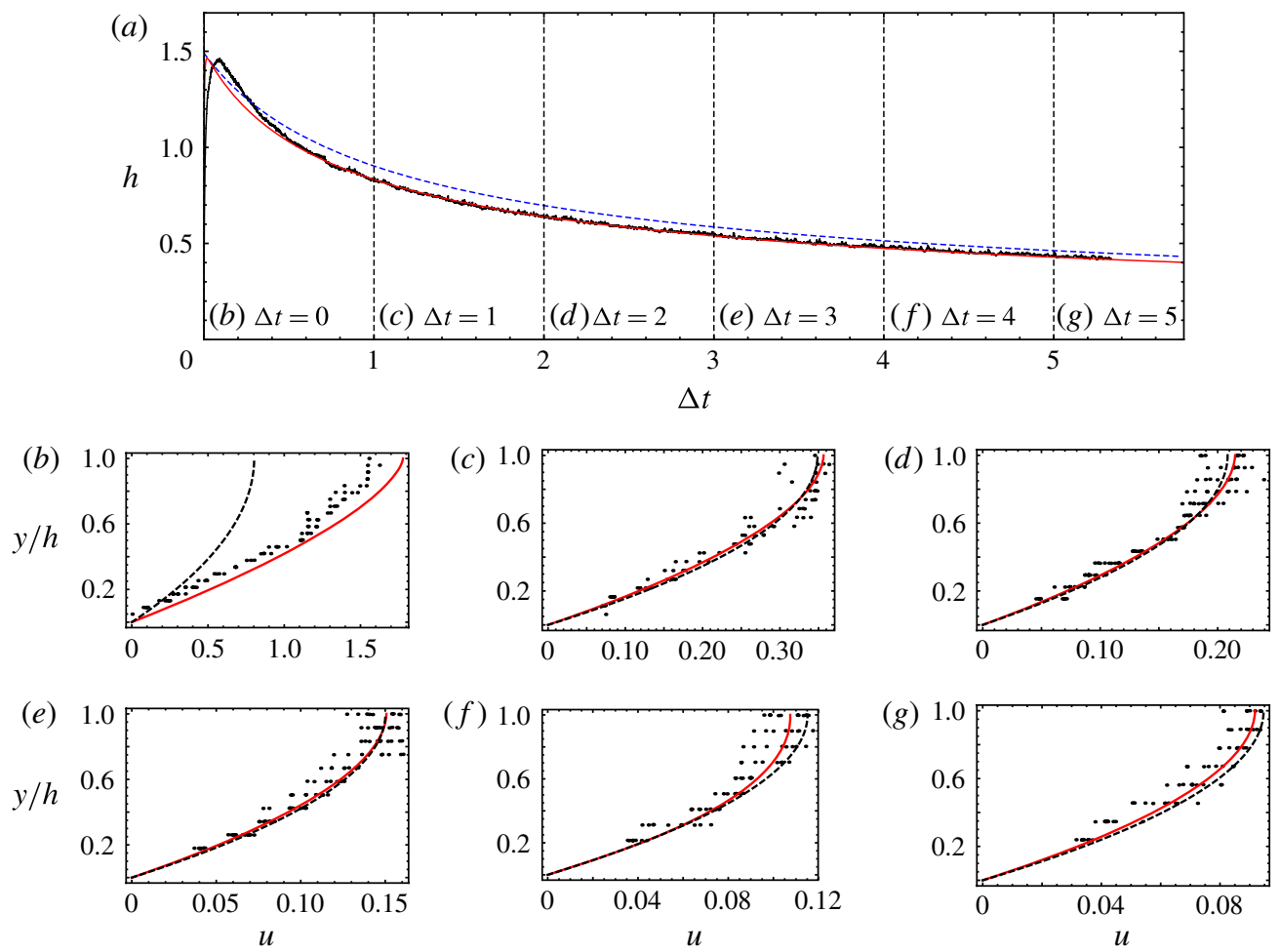

FIGURE 10. (Colour online) (a) Flow depth evolution $h(x, t)$ at $x=1$ and $(b-g)$ velocity profiles taken at different times $\Delta t$ after the front has passed, for run $\mathrm{A}\left(\phi=30 \%, \theta=25^{\circ}\right)$. In panel $(a)$ are plotted the experimental data (black trace), the composite solution (2.7) (solid, red online) and the first-order approximation (2.9) of $h(x, t)$ when sidewall friction is taken into account $\left(\epsilon_{w}=0.099\right)$ (dashed, blue online). In panels $(b-g)$ are plotted the velocity profiles: the dots represent the experimental data, the solid line (red online) is the powerlaw function and the dashed line (grey online) shows the Newtonian profile (2.4). Strictly speaking, the initial velocity profile was not taken at $\Delta t=0$ (as the depth was zero), but at $\Delta t=0.020$ (the depth was $h=1.26$ ). Blunting indices: $(b) n=1.53 \pm 0.06, m=0.57 \pm 0.06$; (c) $n=1.79 \pm 0.07, m=0.74 \pm 0.03$; (d) $n=1.82 \pm 0.05, m=0.74 \pm 0.11$; (e) $n=1.87 \pm 0.06$, $m=0.78 \pm 0.17 ;(f) n=2.18 \pm 0.09, m=0.77 \pm 0.11 ;(g) n=1.92 \pm 0.08, m=0.77 \pm 0.09$.

initial mass $(3 \mathrm{~kg})$ and the same interstitial fluid. For each run, the aspect ratio and the depth-to-width ratio were the same: $\epsilon=4 \times 10^{-3}$ and $\epsilon_{w}=0.09$.

Figures 10, 11, 12 and 13 report the flow depth evolution and velocity profiles for $\phi=30,45,52$ and $57 \%$, respectively. The upper panel $(a)$ of each figure plots the experimental data, the composite $h$ solution (2.7) and the second-order expansion (2.9) for $h$. As the aspect ratio $\epsilon$ was very small, the correction term due to the pressure gradient was very small. In practice, the composite solution could hardly be distinguished from the outer solution $h(1, t)=\sqrt{1 / t}$. As the flow depth curves $h(1, t)$ at $x=1$ did not start at the same time due to the differences in the front position evolution, we shifted all the $h$ curves slightly so that they have the same time origin. In all of these plots, $t=0$ refers to the front arriving at $x=1$ and $\Delta t$ is the time elapsed since the front passed $x=1$. In the lower panels $(b-g)$, we plot the experimental velocity profiles (dots) and the theoretical Newtonian velocity profile (2.4), in which the bulk viscosity law was given by the Krieger-Dougherty 

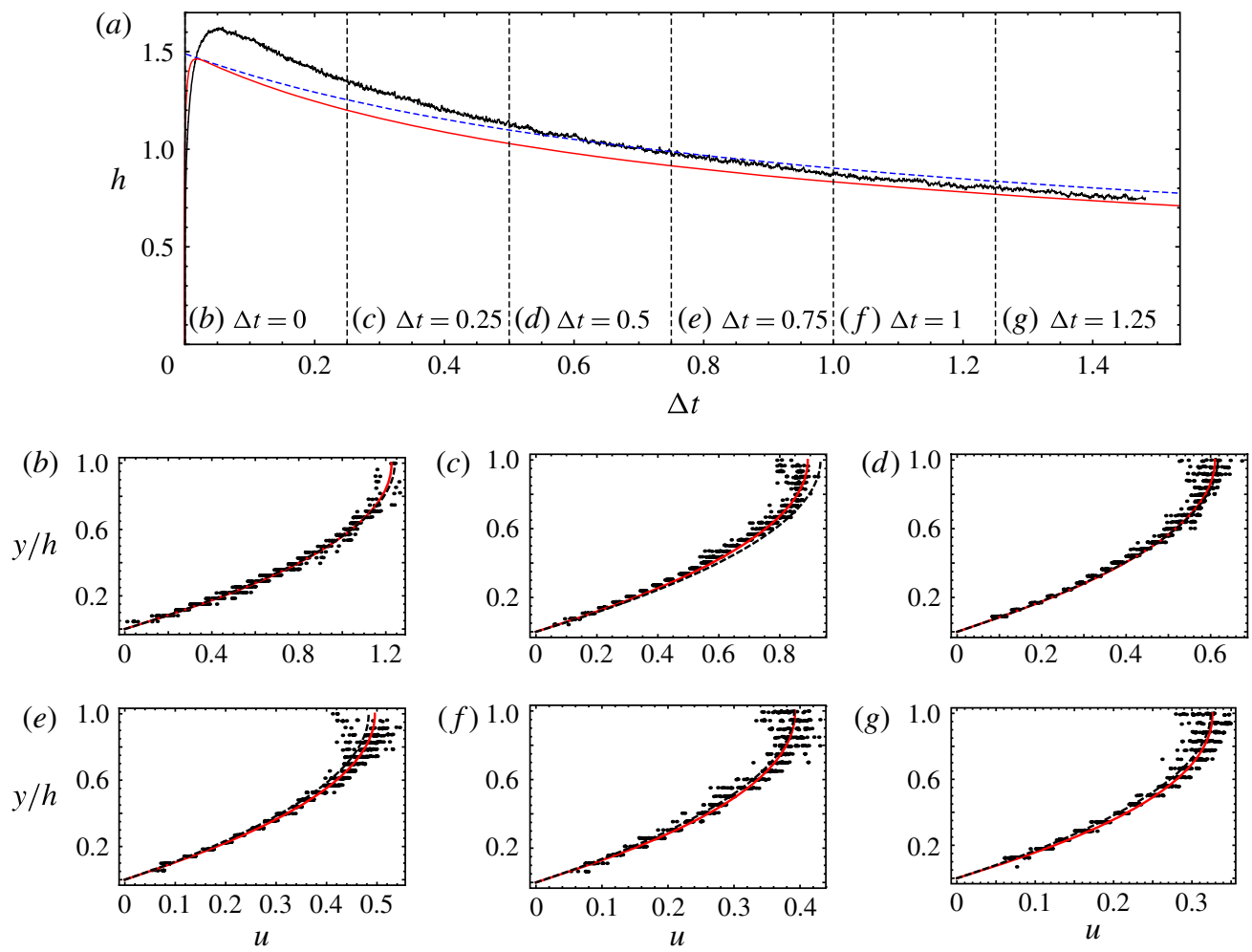

FIgURE 11. (Colour online) (a) Flow depth evolution $h(x, t)$ at $x=1$ and $(b-g)$ velocity profiles taken at different times $\Delta t$ after the front has passed, for run $\mathrm{B}\left(\phi=45 \%, \theta=25^{\circ}\right)$. In panel $(a)$ are plotted the experimental data (black trace), the composite solution (2.7) (solid, red online) and the first-order approximation of $h(x, t)$ when sidewall friction is taken into account $\left(\epsilon_{w}=0.099\right)$ (dashed, blue online). In panels $(b-g)$ are plotted the velocity profiles: the dots represent the experimental data, the solid line (red online) is the power-law function and the dashed line (grey online) shows the Newtonian profile (2.4). Strictly speaking, the initial velocity profile was not taken at $\Delta t=0$, but at $\Delta t=44 \times 10^{-4}$ (the depth was $h=1.23$ ). Blunting indices: $(b) n=2.07 \pm 0.02, m=0.55 \pm 0.01 ;(c) n=2.04 \pm 0.01, m=0.67 \pm 0.05$; (d) $n=2.06 \pm 0.01, m=0.74 \pm 0.05$; (e) $n=2.05 \pm 0.02, m=0.71 \pm 0.05$; $(f) n=2.13 \pm 0.02$, $m=0.73 \pm 0.08 ;(g) n=2.15 \pm 0.02, m=0.75 \pm 0.05$.

relation (2.2) with $\beta=2$ and $\phi_{m}=62.5 \%$. The free-surface gradient was evaluated from the mean slope of the free surface on the images. Note also that for all velocity profiles, we used a scaled coordinate $y / h$ to facilitate comparison of results. To characterize the degree of blunting $n$, we fitted the power-law velocity profile (2.15) on the velocity data. We also computed the non-parametric blunting index (2.16). The respective values of $n$ and $m$ are reported in the captions.

For the lowest concentration (run A, $\phi=30 \%$ ), the flow depth profile was very close to the theoretical profile (2.7) to leading order, as shown by figure $10(a)$. Except for $(b)$, taken just after the front passed, the velocity profiles in figure 10 were parabolic and matched the theoretical profiles (2.4). From this standpoint, the suspension behaved like a homogeneous fluid. The deviation from the parabolic profile seen at the front was also observed with genuinely Newtonian fluids (Andreini et al. 2012). Yet, in contrast to Newtonian fluids, the data showed more noise, especially 

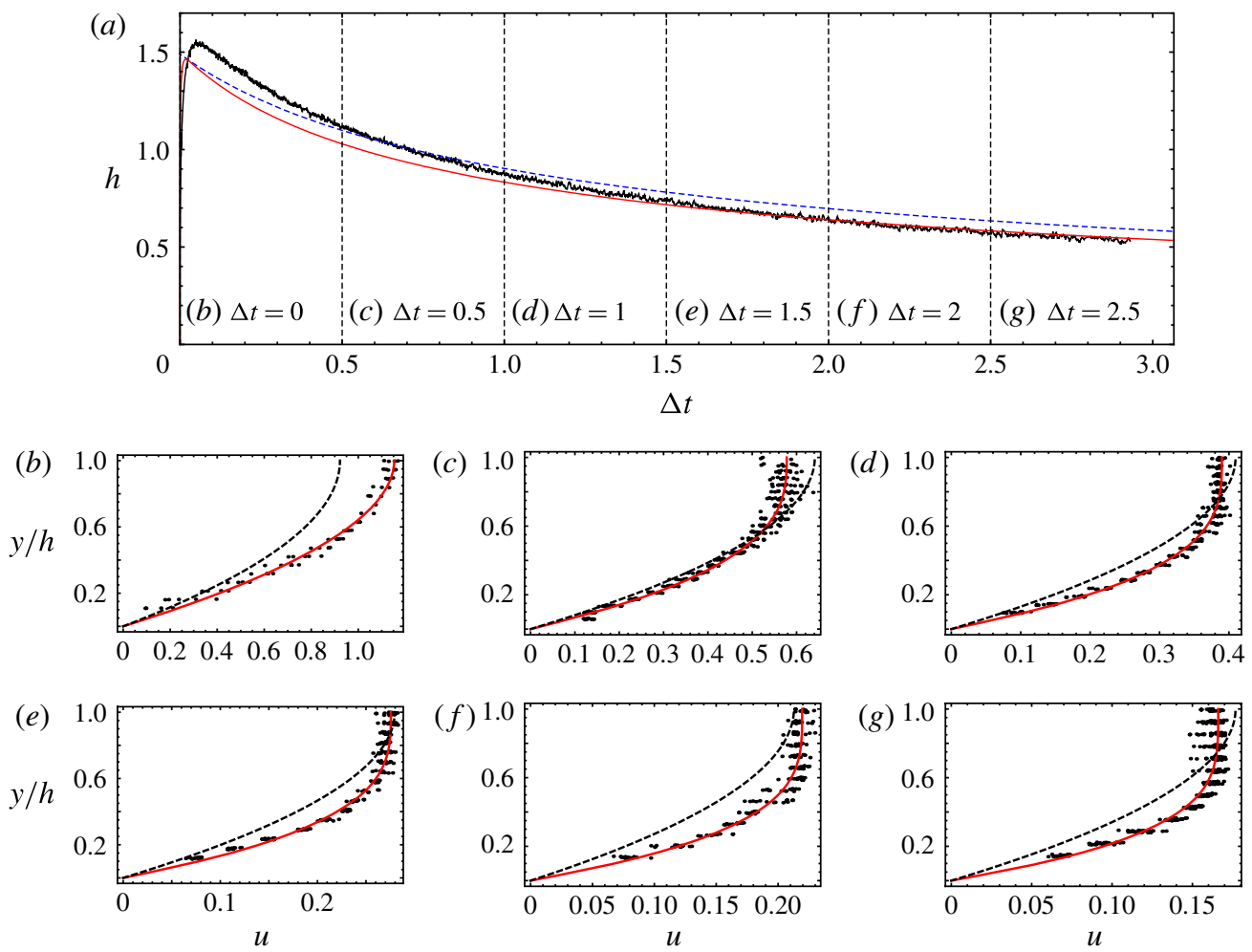

FIgURE 12. (Colour online) (a) Flow depth evolution $h(x, t)$ at $x=1$ and $(b-g)$ velocity profiles taken at different times $\Delta t$ after the front has passed, for run $\mathrm{E}\left(\phi=52 \%, \theta=25^{\circ}\right)$. In panel ( $a$ ) are plotted the experimental data (black trace), the composite solution (2.7) (solid, red online) and the first-order approximation of $h(x, t)$ when sidewall friction is taken into account $\left(\epsilon_{w}=0.099\right)$ (dashed, blue online). In panels $(b-g)$ are plotted the velocity profiles: the dots represent the experimental data, the solid line (red online) is the power-law function and the dashed line (grey online) shows the Newtonian profile (2.4). Strictly speaking, the initial velocity profile was not taken at $\Delta t=0$, but at $\Delta t=5 \times 10^{-4}$. Blunting indices: (b) $n=1.96 \pm 0.03, m=0.69 \pm 0.05$; (c) $n=2.79 \pm 0.03, m=0.77 \pm 0.02$; (d) $n=3.15 \pm 0.03$, $m=0.88 \pm 0.03 ;(e) n=3.15 \pm 0.04, m=0.87 \pm 0.03 ;(f) n=3.51 \pm 0.06, m=0.92 \pm 0.07$; (g) $n=3.76 \pm 0.04, m=0.87 \pm 0.01$.

adjacent to the free surface. As explained in $\S 3.3$, the increase in the noise strength resulted to a large extent from the loss of quality away from the bottom as the number of fluid-particle interfaces increased significantly. Note also the absence of slip at the bottom boundary. Additional videos made with a macro lens confirmed that the suspension had zero velocity relative to the boundary. This was also observed at higher concentrations.

At a moderate concentration (run $\mathrm{B}, \phi=45 \%$ ), we did not observe any apparent change in behaviour: all velocity profiles of figure 11 were parabolic and closely matched the Newtonian profile (2.4). The depth profile closely followed the expected trend (2.7) at sufficiently long times $(t>0.5)$, but, at short times, the flow depth was approximately $10 \%$ higher than predicted by theory. Taking higher-order corrective terms (influence of sidewalls or pressure-induced diffusive effects) in the depth evolution equation (2.7) or (2.9) did not lead to better agreement. This discrepancy 

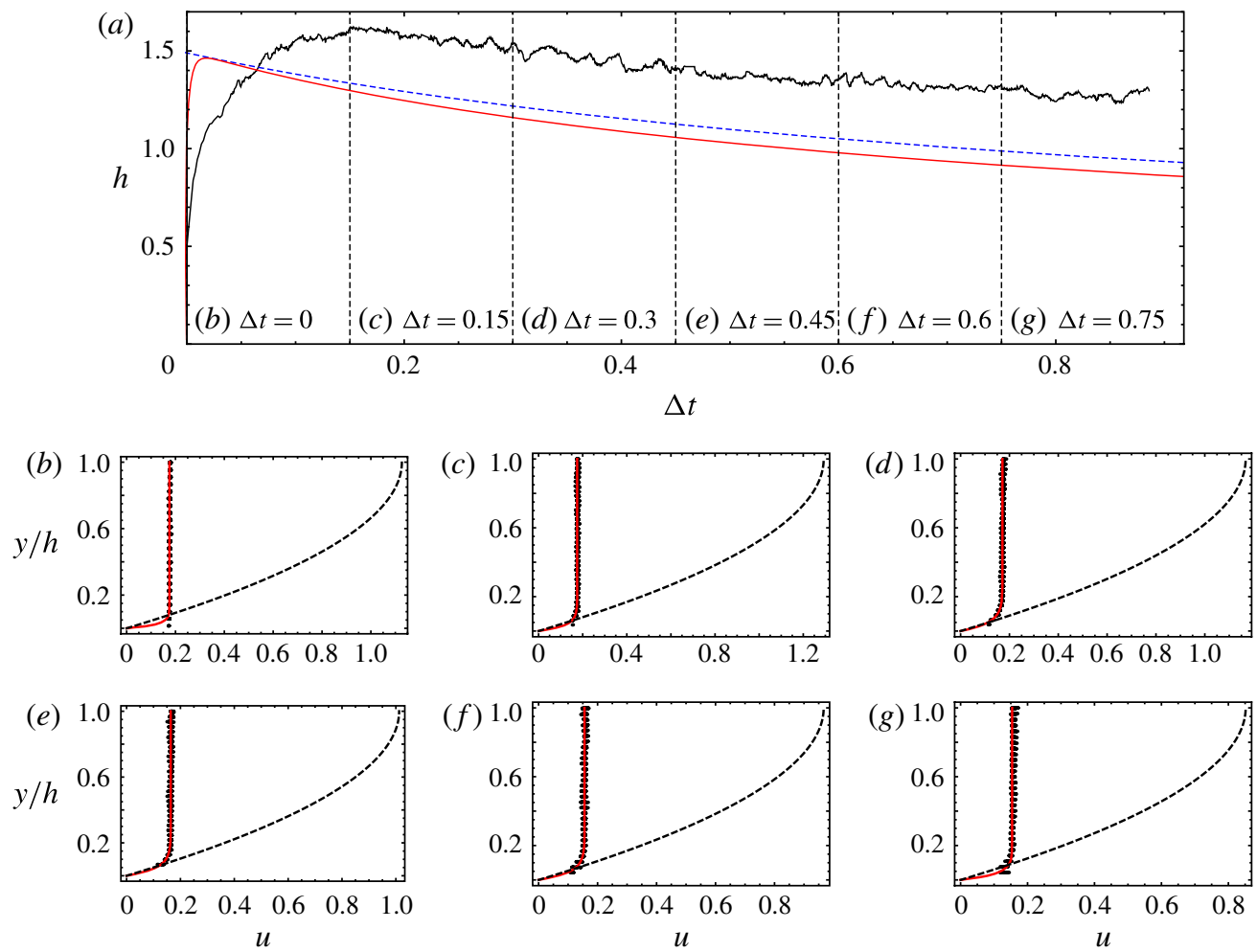

FIgURE 13. (Colour online) (a) Flow depth evolution $h(x, t)$ at $x=1$ and $(b-g)$ velocity profiles taken at different times $\Delta t$ after the front has passed, for run $\mathrm{H}\left(\phi=57 \%, \theta=25^{\circ}\right)$. In panel $(a)$ are plotted the experimental data (black trace), the composite solution (2.7) (solid, red online) and the first-order approximation of $h(x, t)$ when sidewall friction is taken into account $\left(\epsilon_{w}=0.099\right)$ (dashed, blue online). In panels $(b-g)$ are plotted the velocity profiles: the dots represent the experimental data, the solid line (red online) is the power-law function and the dashed line (grey online) shows the Newtonian profile (2.4). Strictly speaking, the initial velocity profile was not taken at $\Delta t=0$, but at $\Delta t=0.01$. Blunting indices: (b) $n=45.7, m=0.99 \pm 0.01$; (c) $n=31.6, m=1.00 \pm 0.01$; (d) $n=20.5, m=0.97 \pm 0.02$; (e) $n=21.1, m=0.97 \pm 0.02$; $(f) n=22.1, m=0.97 \pm 0.03 ;(g) n=39.1, m=0.97 \pm 0.03$.

was, however, sufficiently minor not to question the concordance with the Newtonian model. For both runs A and B, no significant deviation from the Newtonian model was observed, which suggested that particle migration did not occur over the duration of the experiment.

At a higher concentration, here for $\phi=52 \%$ (run E), the differences between the parabolic Newtonian and the experimental profiles were more marked and increased with time. As shown by figure 12, the velocity profile was close to the parabolic shape just behind the head, but, at longer times $(\Delta t \geqslant 1)$, the profile was blunt, as was expected from the particle migration theories outlined in $\S 2.3$. With time, this profile was increasingly blunt, meaning that the flow did not reach steady state over the duration of this run. Surprisingly, the flow depth profile (see figure 12a) was still closely approximated to leading order by the theoretical profile (2.7). Note also that, for this run, the time variations in the front position $x_{f}(t)$ also closely followed the theoretical solution $x_{f}(t)=(9 t / 4)^{1 / 3}$, as shown by figure 9 . This demonstrates 
that the macroscopic properties were well captured by lubrication theory and did not exhibit significant differences compared with those of genuinely Newtonian fluids. Yet, if we take a closer look at the flow properties on the microscopic scale, we see that the material behaviour deviated significantly from that of a Newtonian fluid. Indeed, at long times, we found that the blunting index ranged from 1.96 to 3.76. We can also observe on figure 12 that, while the experimental velocity data were fairly well described using the power-law model (2.15), there was no good correspondence between the two blunting indices. Recall from $\S 2.4$ that, if the velocity profile closely followed the power-law model (2.15), then we should have $m=n /(n+1)$. If we take the experimental profile of figure $12(\mathrm{~g})$, we find that $n /(n+1)=0.79$, whereas the non-parametric blunting index is $m=0.87 \pm 0.01$.

At the highest concentrations, the velocity profiles differed a great deal from the parabolic shape. As shown by figure 13 for run $\mathrm{H}(\phi=57 \%)$, there was a thin layer along the bottom, within which the suspension was sheared, while for $y \geqslant 0.1 h$, the shear rate was very low, but non-zero. This gave rise to a velocity profile whose features were closer to those exhibited by viscoplastic materials than Newtonian fluids. However, the apparent plug flows seemed to be sheared across the whole depth, which would rule out the possibility of a jammed state in the upper flow layers. The low data scatter ( $\pm 3 \%$ for the surface velocity) enabled us to highlight the existence of low shear in the upper layers $(\dot{\gamma} \approx 0.2)$. Moreover, we were successful in adjusting a power-law function (2.15) on the velocity data, and, as shown by figure $13(b-g)$, the power-law model closely matched the experimental data. We found that, very quickly, the non-parametric blunting index $m$ reached a constant value close to 0.97 , but, unlike the previous runs, the index $n$ fluctuated within the 20-40 range (still, this range is consistent with $m=0.97$ as $n /(n+1)$ yields values that range from 0.952 to 0.975). We also noted in figure 13 that the flow was $20 \%$ thicker than a Newtonian fluid with the same bulk viscosity (see figure 13a) except within the leading edge, in which the depth grew much more slowly than for a Newtonian fluid. Experimentally, the maximum flow depth was observed at $\Delta t=0.16$ after the front passed, whereas, theoretically, equation (2.7) obtained using lubrication theory predicts a maximum occurring at $\Delta t=0.017$. Another remarkable feature was that, even within the tip region, the velocity profile was blunt over most of the depth. It is also worth noticing that, although the order of magnitude of the mean velocity predicted by lubrication theory was three times higher than the mean experimental velocity, the Newtonian velocity model seemed to properly capture the velocities in the sheared layer along the bottom.

The flow behaviour and its variations with time are best summarized by looking at the values of the non-parametric blunting index $m$ and the parameter $n$ of the power-law function (2.15) used to fit the velocity profiles. Figure 14(a) shows how $n$ varies as a function of time for all runs, and figure $14(b)$ reports the time variations in $m$. The error bars are also shown, but it is difficult to compare the errors between each representation, as they do not result from the same process. Indeed, for $n$, error bars are the standard errors associated with the fitting of the power-law velocity model (2.15) to the velocity data across the whole depth (the best-fitting curve is that which minimizes the sum of squared residuals). For $m$, the errors mainly represent the standard deviation of the surface velocity (due to the progressive loss of contrast with increasing depth, the velocities at the free surface were more scattered than the mean velocities). This explains why the error bars are longer for $m$ than for $n$.

At moderate concentrations, $n$ was fairly close to 2, which supports our previous conjecture that, on the whole, flow behaviour was nearly Newtonian. The $m$ values 

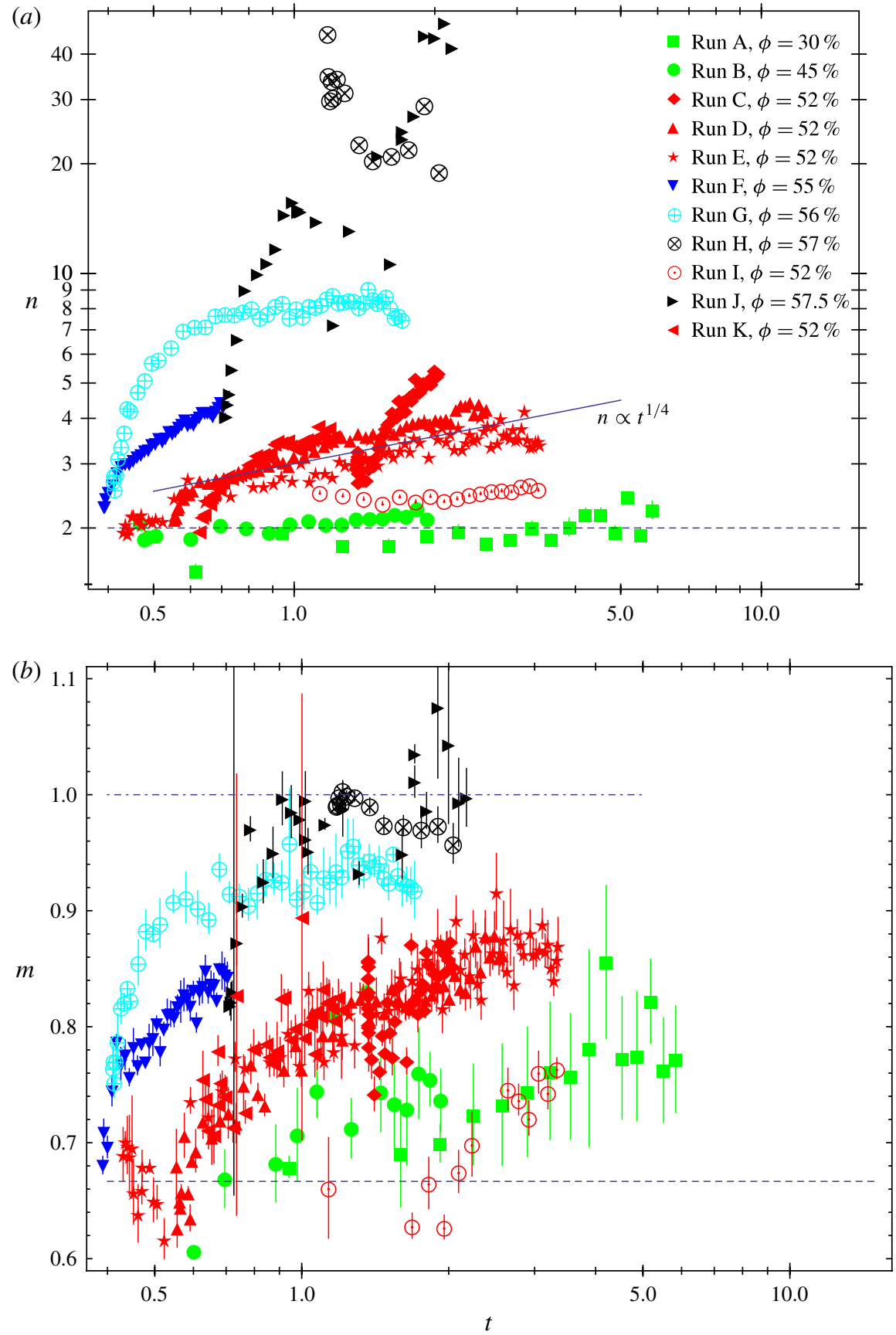

FIGURE 14. (Colour online) (a) Variation of $n$ with time for all runs. The solid line is the empirical trend $3 t^{1 / 4}$; the horizontal dashed line is $n=2$ (Newtonian profile). (b) Evolution of $m$. The two horizontal dashed lines represent the limiting values $2 / 3$ (parabolic profile) and 1 (uniform). 

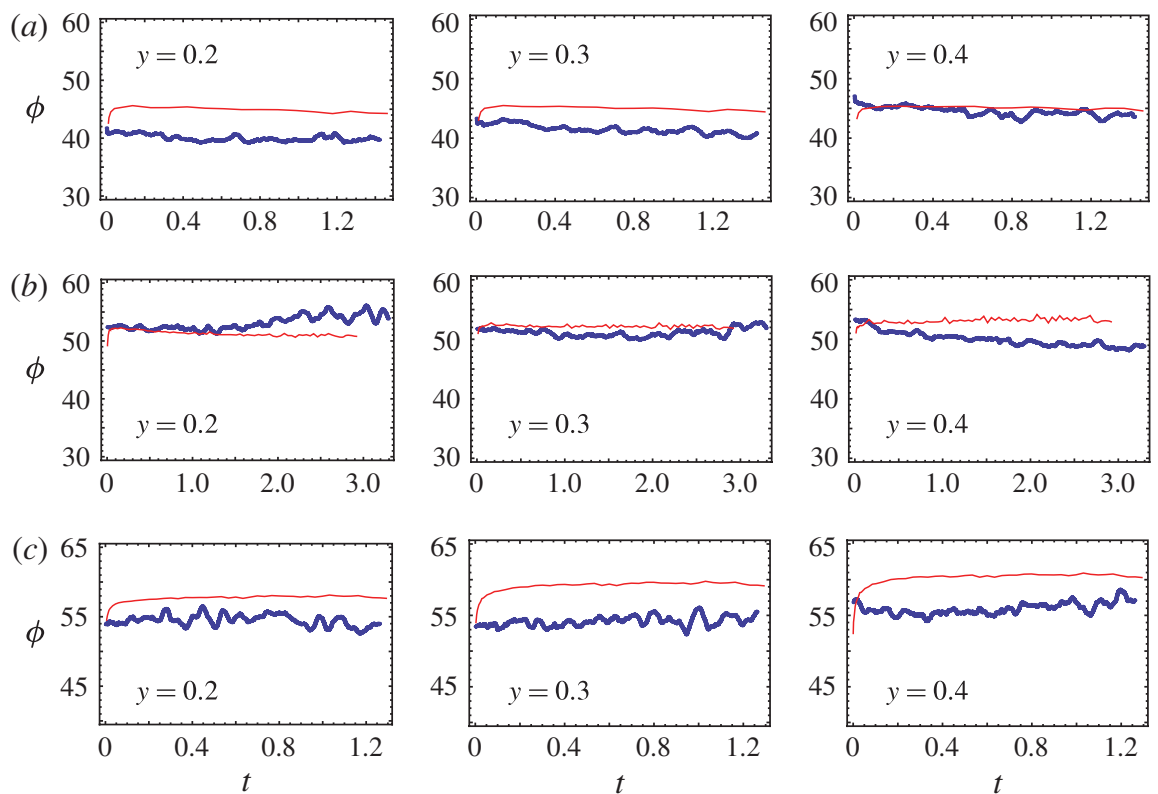

FIGURE 15. (Colour online) Evolution of the particle concentration (thick line) measured at $y=0.2,0.3$ and 0.4 for $(a)$ run $\mathrm{B},(b)$ run $\mathrm{E}$ and $(c)$ run $\mathrm{G}$. The thin curve (red online) represents the theoretical prediction of the particle concentration profile given the velocity profile. The Krieger-Dougherty model of viscosity (2.2) with $\phi_{m}=62.5 \%$ and $\beta=2$ has been used. The uncertainty of the local measurement of $\phi$ was lower than $4 \%$ (see $\S 3.3$ ).

relating to runs $\mathrm{A}$ and $\mathrm{B}$ were, however, significantly different from the Newtonian value $2 / 3$. When increasing the particle concentration, we observed that $n$ continuously increased with time, and, for the highest concentrations, $m$ seemed to stabilize around a constant value close to unity. This convergence to a constant value was not clearcut, as the data were noisy and the run duration was limited. In particular, the $n$ values strongly fluctuated within a wide range, which precludes the conclusive achievement of a steady state. For $\phi<56 \%$, a striking feature is that all data seemed to increase with time according to the same power law as that which characterized the transition regime in the shear-induced migration model (e.g. compare with figure $5 c$ ). For instance, the growth of the $n$ values pertaining to $\phi=52 \%$ was fairly well described using (2.20).

\subsection{Particle concentration}

As we were able to measure the particle concentration in the basal layer by counting the number of particles (see §3.3), we compared these local measurements with the estimates obtained using an inverse method. We assumed that: (i) the Krieger-Dougherty relation (2.2) held true, (ii) the shear stress distribution was given by (2.3), and (iii) the shear rate $\dot{\gamma}$ could be described using the power-law function (2.15) adjusted to experimental data and then differentiating the resulting fitted function with respect to $y$. Figure 15 shows the concentration evolution at $y=0.2,0.3$ and 0.4 for runs B, E and G. For run B $(\bar{\phi}=45 \%)$, there was clearly a concentration gradient near the bottom, with $\phi \sim 40 \%$ at $y=0.2$ and $\phi \sim 45 \%$ at $y=0.4$. This gradient was consistent with particle migration, but contrasted with the 
velocity profiles (see figure 11), which were closely approximated by a Newtonian profile. We also note that the estimated values of $\phi$ exceeded the measured values for $y=0.2$ and 0.3 , but were in close agreement for $y=0.4$. Another key feature was the slow decay of $\phi$ for the three depths $y=0.2,0.3$ and 0.4 , indicating that there was slow migration from the lower to upper layers.

The behaviour of run $\mathrm{E}$ is more difficult to understand. At $y=0.4$, the particle concentration decreased from 54 to $49 \%$ with time, whereas at $y=0.2$ it increased from 52 to $54 \%$. We also note that the theoretical predictions matched the experimental observations at $y=0.2$, but overestimated the concentration at $y=0.4$. In addition to the Krieger-Dougherty relation (2.2), we tested different viscosity functions proposed by Morris \& Boulay (1999), Zarraga et al. (2000) and Boyer et al. (2011). The position of the inverse curves differed from the ones displayed on figure 15, but the agreement did not improve.

In addition to local measurements, we also collected a few samples at the flume outlet at different times. The mean solids fraction was measured by weighing the different components. No variation in the mean particle concentration was detected, which indicated that the particle flux in the $x$-direction, relative to the mean velocity, was negligible. There was no particle accumulation within the leading edge, as is the case for granular flows (Gray \& Ancey 2009).

\section{Concluding remarks}

Let us now put the different elements into perspective by first reviewing the theoretical elements, then looking at how theory compares with experiments. Recall that obtaining a clear theoretical overview of the problem was difficult: indeed, although the governing equations were closed, we were unable to solve the fully coupled problem including the avalanche dynamics and particle migration. Instead, we addressed limiting cases such as particle migration in uniform flows and steady state.

We consider the flow of a fixed volume of concentrated suspension. As initially the suspension is homogeneous, its behaviour should be close to that of a Newtonian fluid, and we expect that the front position varies with time as $x_{f} \propto t^{1 / 3}$, while the flow depth at $x=1$ should vary as $h \propto \sqrt{1 / t}$ (see $\S 2.2$ ). Over time, particles should migrate from the basal layer (high-shear-rate region) towards the free surface (low-shear-rate region). This is a slow process, whose characteristic time (the time taken to reach a steady state) $t_{s s}$ is given by (2.19). However, at high concentrations, this process can be faster (see run H) (Morris \& Brady 1998; Ovarlez, Bertrand \& Rodts 2006). When the run duration is much shorter than $t_{s s}$, we expect that changes in the particle concentration result purely from advection $\left(\partial_{t} \phi+\boldsymbol{u} \nabla \phi=0\right)$, whereas, for run durations much longer than $t_{s s}$, flow reaches a new steady state $(j=0)$, characterized by a steady blunt velocity profile. The degree of blunting, which is represented by the power-law index $n$ in (2.15), ranges from 2.7 to 4 when the mean particle concentration $\bar{\phi}$ is increased from 30 to $60 \%$. For short run durations $D\left(D<t_{s s}\right)$, there is a transition regime, in which the concentration profile becomes gradually less uniform and the velocity profile becomes increasingly more blunt. Typically, the degree of blunting grows as $n \propto t^{p}$, with $p$ ranging from $3.7(\bar{\phi}=30 \%)$ to $7.3(\bar{\phi}=60 \%)$ (see $\left.\S 2.4\right)$. A direct consequence of particle migration is flow acceleration. Indeed, as the velocity profile becomes blunter, the viscosity drops in the basal layer and increases in the upper layer. Shear-induced diffusion theory predicts that the mean velocity could be increased by a factor of more than two (e.g. see figure 5). If so, we then expect that the front position 
exceeds that of a well-mixed homogeneous suspension with the same solids fraction. Mass conservation implies that the flow depth should be thinner.

The experiments presented in $\S 4$ show that the theoretical picture drawn above is partially correct. First, let us take a look at the macroscopic features: the flow depth and front position evolutions. Figure 9 shows that the theoretical prediction $x_{f} \propto t^{1 / 3}$ was in good agreement with data, especially at moderate concentrations $(\bar{\phi} \leqslant 45 \%)$, but, for the highest concentrations $(\bar{\phi} \geqslant 52 \%)$, the front position varied as $x_{f} \propto t^{0.3}$ instead, and there was a systematic delay, which could not be explained solely by sidewall friction. Although the scaling relation was still fairly accurate, relative differences as large as $50 \%$ could be observed. If lubrication theory were correct, this time should be close to $4 / 9 \approx 0.44$ according to $(2.6)$; if sidewall friction is accounted for, this time is approximately 0.47 . For runs $\mathrm{B}\left(t_{0}=0.46\right), \mathrm{E}\left(t_{0}=0.43\right), \mathrm{F}\left(t_{0}=0.38\right)$ and $\mathrm{G}\left(t_{0}=0.41\right)$, the front arrived at $x=1$ earlier than predicted by lubrication theory, confirming that flow acceleration occurred. This acceleration was, however, moderate, as $t_{0}$ was very close to the theoretical value 4/9 except for run F. For other runs, the front arrived late. The maximum delay was observed with the highest concentrations (runs $\mathrm{H}$ and $\mathrm{J}$, respectively, $t_{0}=1.17$ and 0.71 ) or the shallowest slopes (runs $\mathrm{C}$ and $\mathrm{D}$, respectively, $t_{0}=1.37$ and 0.55 ). Slope influence (compare runs $\mathrm{C}, \mathrm{D}$ and $\mathrm{E}$ ) is rather strange, as it was not observed for homogeneous Newtonian fluids (Andreini et al. 2012). It may be an indication of shear-stress dependence as a result of physical contact between particles. As we also note that these runs corresponded to the lowest velocities (when expressed in physical units), another possible explanation is that the viscous scaling in our analytical treatment is incorrect, at least for slow flows. In contrast, the flow depth profile was fairly well captured by lubrication theory except for the highest concentrations, for which the mismatch of $h$ reached $20 \%$.

Now let us address the microscopic features. All run durations were shorter than the (theoretical) time to convergence $t_{s s}$. For this reason, we should not expect to observe steady states in our experimental results. Figure 14 shows how the degree of blunting varied with time for all of our runs. For a number of these runs, the growth rate of $n$ was consistent with the predictions of the shear-induced diffusion model. There were, however, noticeable exceptions (for runs $\mathrm{H}$ and $\mathrm{J}$, there was no clear trend). In $\S 2.3$, we have shown that migration theory leads to values of $n$ in the 2.4-6.0 range for the steady-state regime (see figure 3). As the run duration was not sufficient to reach steady state, it is difficult to compare theory and experiments on this particular point, but at least we notice that the predicted values are consistent with measurements in the transition regime for $\bar{\phi}<56 \%$. For $\bar{\phi}$ in excess of $56 \%$, the velocity index $n$ lies in the 4-40 range, which leads to the conclusion that the steady-state value is likely to be much larger than the theoretical value (see figure 14). As we were unable to measure the concentration profiles throughout the whole depth, it was difficult to be more conclusive on the performance of the model in its ability to predict the concentration changes over time. Quantitative comparison of the concentration evolution near the bottom showed partial agreement.

In contrast with most earlier investigations, we used slightly polydisperse suspensions with a continuous size distribution. Previous studies performed with bimodal distributions showed that the finest particles were more uniformly distributed throughout the depth whereas the coarsest particles accumulated in low-shear regions (Krishnan et al. 1996; Lyon \& Leal 1998b; Hsiao et al. 2005). This enrichment in coarse particles reflected flow-induced size segregation and was consistent with the particle size dependence of the migration velocity of the diffusive flux model 
except for the $a^{p}$ dependence, where $p=2.6-2.9$ rather than $a^{2}$ (Abbott $e t$ al. 1991; Tetlow et al. 1998; Hsiao et al. 2005). The effects of polydispersity on the rheological behaviour of particle suspensions are poorly known. One documented effect is viscosity reduction, largely attributed to the increase in the maximum packing fraction: the bulk viscosities of polydisperse suspensions are correspondingly lower than for their monodisperse counterparts at the same total solids fraction (Cheng 1984; Gondret \& Petit 1997). Another effect may be shear thinning (Zarraga et al. 2000; Stickel \& Powell 2005), regardless of whether it is real or apparent. Reardon et al. (2008) reported on falling-ball experiments. They observed that, for solids fractions in excess of $50 \%$, the bulk viscosity exhibited a shear-rate dependence, whose strength was conditioned by the degree of polydispersity. When the ratio of the standard deviation to the mean diameter was 0.3 (like for the samples we used), they found that the shear-thinning index was close to 0.6. From this perspective, it is difficult to distinguish between the apparent shear thinning induced by particle migration and that due to polydispersity. The last possible effect is size segregation. For dry granular flows, polydispersity reduces size segregation to a large extent (Gray 2010). For this reason, we think that size segregation was unlikely to produce significant effects here, but this should be confirmed by further investigations.

With our local measurements, we come to conclusions that differ from those drawn by Bonnoit et al. (2010). On the basis of macroscopic measurements on gravity-driven flows down an inclined plane, these authors concluded that the bulk behaved like a Newtonian fluid for solids fractions as large as $61 \%$. In our experiments, for concentrations in excess of $52 \%$, there was clear evidence that the internal dynamics was richer than what the bulk measurements showed. As illustrated by run E (see figures 9 and 12), the suspension behaved as a Newtonian fluid from a macroscopic viewpoint, but the velocity profiles demonstrated that the suspension underwent particle migration, which altered the rheology of the suspension. This difference in the determination of the rheology from macroscopic and local measurements is not new, as it has been documented for Couette cells (Ovarlez et al. 2006). Interestingly, one argument used by Bonnoit et al. (2010) to exclude the possibility of particle migration was to estimate the blunting index $n$ from velocity measurements at the leading edge, but we observed that the blunting index was close to 2 within the leading edge, then grew more or less abruptly away from the front (see figure 14). This probably resulted from the intense mixing within the tip region. Another difference from the conclusions of Bonnoit et al. (2010) is that we started to observe deviations from the Newtonian behaviour at the bulk scale for concentrations in excess of $60 \%$, whereas they did not see any change for concentrations up to $61 \%$. One possible explanation is that they used slightly positively buoyant particles $\left(\Delta \rho=10-20 \mathrm{~kg} \mathrm{~m}^{-3}\right)$ and this may have been sufficient to counterbalance the effects of particle migration, at least at short times.

A recurrent feature of the velocity profiles presented here is the absence of slip at the lower solid boundary, to within experimental uncertainty. A few authors such as Koh, Hookham \& Leal (1994) and more recently Medhi, Kumar \& Singh (2011) observed a significant slip between the particles and the carrier fluid near a solid boundary. As Koh et al. (1994) themselves admitted, the relative motion of the particle and fluid phases is difficult to understand if the density mismatches are vanishingly small. This issue clearly merits further investigations. 


\section{Acknowledgements}

The work presented here was supported by the Swiss National Science Foundation under grant number 200021-105193/1 (a project called 'Transient free-surface flows of concentrated suspensions. Application to geophysical flows', funded by an R'Equip grant), the Competence Centre in Mobile Information and Communication Systems (a centre supported by the Swiss National Science Foundation under grant number 500567322, MICS project), the Competence Centre in Environmental Sciences (TRAMM and APUNCH projects), and specific funds provided by EPFL (Vice-Présidence à la Recherche). We are grateful to $\mathrm{B}$. Bates for improving the English in the manuscript and to the reviewers, whose constructive remarks helped to improve the paper significantly.

\section{Supplementary material and movies}

Supplementary material and movies are available at http://dx.doi.org/10.1017/jfm. 2013.154.

\section{REFERENCES}

Aвbott, J. R., Tetlow, N., Graham, A. L., Altobelli, S. A., Fukushima, E., Mondy, L. A. \& STEPhens, T. S. 1991 Experimental observations of particle migration in concentrated suspensions: Couette flow. J. Rheol. 35, 773-795.

ANCEY, C. 2012 Gravity flow on steep slope. In Buoyancy Driven Flows (ed. E. Chassignet, C. Cenedese \& J. Verron), Cambridge University Press.

Ancey, C., Andreini, N. \& Epely-Chauvin, G. 2013 Granular suspensions I. Macro-viscous behaviour. Phys. Fluids 25, 033301.

Ancey, C., Cochard, S. \& ANDreini, N. 2009 The dam-break problem for viscous fluids in the high-capillary-number limit. J. Fluid Mech. 624, 1-22.

ANDREINI, N. 2012 Dam break of Newtonian fluids and granular suspensions: internal dynamics measurements. PhD thesis, École Polytechnique Fédérale de Lausanne.

Andreini, N., Ancey, C. \& Epely-Chauvin, G. 2013 Granular suspensions. II. Plastic regime. Phys. Fluids 25, 033302.

Andreini, N., Epely-Chauvin, G. \& AnCEy, C. 2012 Internal dynamics of Newtonian and viscoplastic fluid avalanches down a sloping bed. Phys. Fluids 24, 053101.

Bonnoit, C., Darnige, T., Clément, E. \& Lindner, A. 2010 Inclined plane rheometry of a dense granular suspension. J. Rheol. 54, 65-79.

Boyer, F., Guazzelli, E. \& Pouliquen, O. 2011 Unifying suspension and granular rheology. Phys. Rev. Lett. 107, 188301.

Chang, C. \& Powell, R. L. 1994 Effect of particle size distributions on the rheology of concentrated bimodal suspensions. J. Rheol. 38, 85-98.

Cheng, D. C. H. 1984 Further observations on the rheological behaviour of dense suspensions. Powder Technol. 37, 255-273.

Cook, P. E., Bertozzi, A. L. \& Hosoi, A. E. 2008 Shock solutions for particle-laden thin films. SIAM J. Appl. Maths 68, 760-783.

Gondret, P. \& Petit, L. 1997 Dynamic viscosity of macroscopic suspensions of bimodal sized solid spheres. J. Rheol. 41, 1261-1274.

GRAY, J. M. N. T. 2010 Particle size segregation in granular avalanches: a brief review of recent progress. In AIP Conference Proceedings, vol. 1227 (ed. J. D. Goddard, J. T. Jenkins \& P. Giovine). pp. 343-362. American Institute of Physics.

GRAY, J. M. N. T. \& ANCEY, C. 2009 Segregation, recirculation and deposition of coarse particles near two-dimensional avalanche fronts. J. Fluid Mech. 629, 387-423.

Hansen, F. K. \& Rødsrud, G. 1991 Surface tension by pendant drop: I. A fast standard instrument using computer image analysis. J. Colloid Interface Sci. 141, 1-9. 
Hsiao, S. C., Christensen, D., Ingber, M. S., Mondy, L. A. \& Altobelli, S. A. 2005 Particle migration rates in a Couette apparatus. J. Mech. 21, 71-75.

HUPPERT, H. E. 1982a Flow and instability of a viscous current down a slope. Nature 300, 427-429.

HUPPERT, H. E. $1982 b$ The propagation of two-dimensional and axisymmetric viscous gravity currents over a rigid horizontal surface. J. Fluid Mech. 121, 43-58.

Koн, C. J., Hоокнам, P. \& Leal, L. G. 1994 An experimental investigation of concentrated suspension flows in a rectangular channel. J. Fluid Mech. 266, 1-32.

Krieger, I. M. \& DougherTy, T. J. 1959 A mechanism for non-Newtonian flow in suspensions of rigid spheres. Trans. Soc. Rheol. 3, 137-152.

Krishnan, G. P., Beimfohr, S. \& Leighton, D. T. 1996 Shear-induced radial segregations in bidisperse suspensions. J. Fluid Mech. 321, 371-393.

Leighton, D. \& ACRivos, A. 1987 The shear-induced migration of particles in concentrated suspensions. J. Fluid Mech. 181, 415-439.

Lhuillier, D. 2009 Migration of rigid particles in non-Brownian viscous suspensions. Phys. Fluids 21, 023302.

LYON, M. K. \& LEAL, L. G. $1998 a$ An experimental study of the motion of concentrated suspensions in two-dimensional channel flow. Part 1. Monodisperse systems. J. Fluid Mech. $363,25-56$.

LYON, M. K. \& LEAL, L. G. $1998 b$ An experimental study of the motion of concentrated suspensions in two-dimensional channel flow. Part 2. Bidisperse systems. J. Fluid Mech. 363, $57-77$.

Medhi, B. J., Kumar, A. \& Singh, A. 2011 Apparent wall slip velocity measurements in free surface flow of concentrated suspensions. Intl J. Multiphase Flow 37, 609-619.

MORRIS, J. F. 2009 A review of microstructure in concentrated suspensions and its implications for rheology and bulk flow. Rheol. Acta 48, 909-923.

MorRis, J. F. \& Boulay, F. 1999 Curvilinear flows of noncolloidal suspensions: the role of normal stresses. J. Rheol. 43, 1213-1238.

MorRis, J. F. \& BRADY, J. F. 1998 Pressure-driven flow of a suspension: buoyancy effects. Intl J. Multiphase Flow 24, 105-130.

Norman, J. T., NAyak, H. V. \& Bonnecaze, R. T. 2005 Migration of buoyant particles in low-Reynolds-number pressure-driven flows. J. Fluid Mech. 523, 1-35.

NotT, P. R. \& BRADY, J. F 1994 Pressure-driven flow of suspensions: simulation and theory. J. Fluid Mech. 275, 157-199.

Nott, P. R., Guazzelli, E. \& Pouliquen, O. 2011 The suspension balance model revisited. Phys. Fluids 23, 043304.

Nsom, B. 2000 The dam break problem for a hyperconcentrated suspension. Appl. Rheol. 10, 224-230.

Ovarlez, G., Bertrand, F. \& Rodts, S. 2006 Local determination of the constitutive law of a dense suspension of noncolloidal particles through magnetic resonance imaging. J. Rheol. 50, 259-292.

Phillips, R. J., Armstrong, R. C., Brown, R. A., Graham, A. L. \& Abbott, J. R. 1992 A constitutive equation for concentrated suspensions that accounts for shear-induced particle migration. Phys. Fluids A 4, 30-39.

Raffel, M., Willert, C. E., Wereley, S. T. \& Kompenhans, J. 2007 Particle Image Velocimetry. Springer.

Reardon, P. T., Feng, S., Graham, A. L., Chawla, V., Admuthe, R. S. \& Abbott, J. R. 2008 Shear-thinning of polydisperse suspensions. J. Phys. D: Appl. Phys. 41, 115408.

Stickel, J. J. \& Powell, R. L. 2005 Fluid mechanics and rheology of dense suspensions. Annu. Rev. Fluid Mech. 37, 129-149.

SVeen, J. K. 2004 An introduction to MatPIV. In Mechanics and Applied Mathematics. Tech Rep. eprint series No. 2. Department of Mathematics, University of Oslo.

Tetlow, N., Graham, A. L., Ingber, M. S., Subia, S. R., Mondy, L. A. \& Altobelli, S. A. 1998 Particle migration in a Couette apparatus: experiment and modelling. J. Rheol. 42, 307-328. 
Timberlake, B. D. \& Morris, J. F. 2005 Particle migration and free-surface topography in inclined plane flow of a suspension. J. Fluid Mech. 538, 309-341.

Ward, T., Wey, C., Gidden, R., Hosoi, A. E. \& Bertozzi, A. L. 2009 Experimental study of gravitation effects in the flow of a particle-laden thin film on an inclined plane. Phys. Fluids 21, 083305.

Wiederseiner, S., Andreini, N., Epely-Chauvin, G. \& Ancey, C. 2011 Refractive index matching in concentrated particle suspensions: a review. Exp. Fluids 50, 1183-1206.

Zarraga, I. E., Hill, D. A. \& Leighton, D. T. 2000 The characterization of the total stress of concentrated suspensions of noncolloidal spheres in Newtonian fluids. J. Rheol. 44, 185-221. 